\title{
LOOP TYPE SUBCONTINUA OF POSITIVE SOLUTIONS FOR INDEFINITE CONCAVE-CONVEX PROBLEMS
}

\author{
URIEL KAUFMANN, HUMBERTO RAMOS QUOIRIN, AND KENICHIRO UMEZU
}

\begin{abstract}
We establish the existence of loop type subcontinua of nonnegative solutions for a class of concave-convex type elliptic equations with indefinite weights, under Dirichlet and Neumann boundary conditions. Our approach depends on local and global bifurcation analysis from the zero solution in a non-regular setting, since the nonlinearities considered are not differentiable at zero, so that the standard bifurcation theory does not apply. To overcome this difficulty, we combine a regularization scheme with a priori bounds, and Whyburn's topological method. Furthermore, via a continuity argument we prove a positivity property for subcontinua of nonnegative solutions. These results are based on a positivity theorem for the associated concave problem proved in [15], and extend previous results established in the powerlike case.
\end{abstract}

\section{IntRoduction AND MAIN RESUlts}

Let $\Omega \subset \mathbb{R}^{N}(N \geq 1)$ be a bounded domain with smooth boundary $\partial \Omega$. In this paper, we consider nonnegative solutions of the problem

$$
\begin{cases}-\Delta u=\lambda a(x) f(u)+b(x) g(u) & \text { in } \Omega, \\ \mathcal{B} u=0 & \text { on } \partial \Omega\end{cases}
$$

where:

- $\Delta$ is the usual Laplacian in $\mathbb{R}^{N}$;

- $\mathcal{B} u:=u$ (Dirichlet) or $\mathcal{B} u:=\frac{\partial u}{\partial \mathbf{n}}$ (Neumann), where $\mathbf{n}$ is the outward unit normal to $\partial \Omega$;

- $\lambda \in \mathbb{R}$ is a bifurcation parameter;

- $a, b \in C(\bar{\Omega})$ are such that $a$ changes sign in $\Omega$ and $b\left(x_{0}\right)>0$ for some $x_{0} \in \Omega$

- $f, g:[0, \infty) \rightarrow \mathbb{R}$ are continuous functions with $f(0)=g(0)=0$.

It follows that $\left(P_{\mathcal{B}}\right)$ possesses the trivial line $(\lambda, 0)$ of zero solutions. The prototype of $f, g$ to be considered in this paper is

$$
f(s)=s^{q}, \quad g(s)=s^{p}, \quad \text { with } 0<q<1<p,
$$

2010 Mathematics Subject Classification. 35J25, 35J61, 35B32.

Key words and phrases. Concave-convex problem; Positive solution; Indefinite nonlinearity; Loop bifurcation.

U. Kaufmann was partially supported by Secyt-UNC 30720150100019CB.

H. Ramos Quoirin was supported by FONDECYT grants 1161635, 1171532 and 1171691.

K. Umezu was supported by JSPS KAKENHI Grant Numbers 15K04945 and 18K03353. 
so that the nonlinearity $\lambda f(s)+g(s)$ has a concave-convex nature. More precisely, we assume that $f \in C^{1}((0, \infty))$ with $f(s)>0$ for $s>0$ and $g \in C^{1}([0, \infty))$ satisfy

$$
\begin{aligned}
& \lim _{s \rightarrow 0^{+}} \frac{f(s)}{s}=\infty, \\
& \lim _{s \rightarrow 0^{+}} \frac{g(s)}{s}=0, \\
& \lim _{s \rightarrow \infty} \frac{f(s)}{s}=0, \\
& \lim _{s \rightarrow \infty} \frac{g(s)}{s}=\infty .
\end{aligned}
$$

Let $r>N$. A function $u \in W^{2, r}(\Omega)$ (and consequently, $u \in C^{1}(\bar{\Omega})$ ) is said to be a nonnegative solution of $\left(P_{\mathcal{B}}\right)$ if $u \geq 0$ in $\Omega, u$ satisfies the equation pointwisely a.e. in $\Omega$, and $\mathcal{B} u=0$ on $\partial \Omega$. If, in addition, $u$ satisfies

$$
\begin{cases}u>0 \text { in } \Omega \text { and } \frac{\partial u}{\partial \mathbf{n}}<0 \text { on } \partial \Omega & \text { if } \mathcal{B} u=u, \\ u>0 \text { on } \bar{\Omega} & \text { if } \mathcal{B} u=\frac{\partial u}{\partial \mathbf{n}},\end{cases}
$$

then we write $u \gg 0$. In this case $u$ lies in the interior of the positive cone $\{u \in$ $V: u \geq 0\}$, where

$$
V:= \begin{cases}C_{0}^{1}(\bar{\Omega}):=\left\{u \in C^{1}(\bar{\Omega}): u=0 \text { on } \partial \Omega\right\} & \text { if } \quad \mathcal{B} u=u, \\ C^{1}(\bar{\Omega}) & \text { if } \quad \mathcal{B} u=\frac{\partial u}{\partial \mathbf{n}} .\end{cases}
$$

A nonnegative solution $u$ of $\left(P_{\mathcal{B}}\right)$ is called positive if $u \gg 0$.

Our first goal is to establish, under certain conditions on $a$ and $b$, the existence of loop type subcontinua $\{(\lambda, u)\}$ (i.e., nonempty, closed and connected subsets in $\mathbb{R} \times V)$ composed by $(0,0)$ and nontrivial nonnegative solutions $(\lambda, u)$ of $\left(P_{\mathcal{B}}\right)$. We shall prove the existence of a loop type subcontinuum $\mathcal{C}_{0}$ such that

$$
\mathcal{C}_{0} \cap\{(\lambda, 0): \lambda \in \mathbb{R}\}=\{(0,0)\} .
$$

It should be emphasized that, in general, one can not deduce that nontrivial nonnegative solutions of $\left(P_{\mathcal{B}}\right)$ satisfy $u \gg 0$, since the strong maximum principle does not apply. This is due to the fact that $a(x) f(\cdot)$ does not satisfy the slope condition [1, p.623], see Remark 2.1(ii) below. As a matter of fact, $\left(P_{\mathcal{B}}\right)$ may have solutions $u$ satisfying $u>0$ in $\Omega$ but not $u \gg 0$ (for concrete examples one may argue as in the proof of [15, Proposition 2.9] after a slight modification). In view of this difficulty, our second purpose is to show that nontrivial solutions lying on $\mathcal{C}_{0}$ satisfy $u \gg 0$.

Bounded subcontinua of positive solutions for indefinite superlinear equations of the form

$$
-\Delta u=\lambda a(x) u+b(x) u^{p} \quad \text { in } \Omega,
$$

with $\Omega$ bounded (under different boundary conditions) or $\Omega=\mathbb{R}^{N}$, have been studied by several authors, see e.g. [4, 5, 8, 6, 22, 7, 20, 19. According to [6, 7, 20, a bounded subcontinuum linking two different points on $(\lambda, 0)$ is called a mushroom, one that meets a single point on $(\lambda, 0)$ is called a loop, and one that does not touch 
$(\lambda, 0)$ is called an isola. Cingolani and Gámez studied both the Dirichlet condition case and the case $\Omega=\mathbb{R}^{N}$, proving the existence of mushrooms $[8$, Theorems 4.4 and 5.5]. Cano-Casanova considered a mixed boundary condition (with a second order uniformly strongly elliptic operator), and proved the existence of a mushroom [5, Theorem 1.4]. López-Gómez and Molina-Meyer dealt with the Dirichlet condition, and established existence results for a mushroom, a loop and an isola in three cases, respectively [20, Theorems 3.1, 5.1 and 5.2]. In the case of Neumann boundary conditions, Brown proved the existence of a mushroom and a loop in two situations, respectively [4, Sections 2 and 5]. Finally, we refer to [27, Section 3] for the existence of a mushroom of positive solutions for a semilinear elliptic problem with a logistic nonlinearity and an indefinite weight, coupled with a nonlinear boundary condition. Let us emphasize that all the previous works hold in the regular case, i.e., when the nonlinearity considered is $C^{1}$ at $u=0$, so that the general theory on local and global bifurcation from simple eigenvalues can be directly applied.

Regarding existence results for positive solutions of concave-convex problems, a large number of works have been devoted to $\left(P_{\mathcal{B}}\right)$ in the 'definite case' (i.e. with $a \geq 0, a \not \equiv 0)$ since the classical work of Ambrosetti, Brezis and Cerami [3], which treats the model case (1.1) with $a=b \equiv 1$ and $p \leq \frac{N+2}{N-2}$ under the Dirichlet boundary condition. In [3] it is proved that $\left(P_{\mathcal{B}}\right)$ has two positive solutions for $\lambda>0$ sufficiently small. This result was extended by De Figueiredo, Gossez and Ubilla [1] to the non-powerlike case, with $a \geq 0$. In addition, in [10, the authors allowed $a$ to change sign and proved the existence of two nontrivial nonnegative solutions of $\left(P_{\mathcal{B}}\right)$ for $\lambda>0$ small. We refer to [24 for a discussion on concaveconvex problems under the Neumann boundary condition.

To the best of our knowledge, besides [15, 24] there are no works providing the existence of solutions that are positive in $\Omega$ for indefinite concave-convex problems (i.e., with $a$ changing sign). In [15, 16, 17] we first established a positivity property for $\left(P_{\mathcal{B}}\right)$ in the powerlike and concave case, i.e. with $f(s)=s^{q}$ and $b \equiv 0$. Thanks to these results, we obtained a positivity result for $\left(P_{\mathcal{B}}\right)$ with $f(s)=s^{q}$ and $b \equiv 1$ (see [15. Section 4]). Finally, let us mention that in the model case (1.1), the existence of a loop type subcontinuum of nonnegative solutions for the Neumann case was obtained by means of a bifurcation approach in 24]. Furthermore, the asymptotic profile of nonnegative solutions as $\lambda \rightarrow 0^{+}$enables one to deduce in some cases their positivity for $\lambda>0$ small, cf. [24, Corollary 1.3].

For our first purpose, we assume that there exist two balls $B, B^{\prime} \Subset \Omega$ and constants $a_{0}, a_{0}^{\prime}, b_{0}, b_{0}^{\prime}>0$ such that

$$
\begin{cases}a(x) \geq a_{0} \text { and } b(x) \geq b_{0} & \text { in } B \\ -a(x) \geq a_{0}^{\prime} \text { and } b(x) \geq b_{0}^{\prime} & \text { in } B^{\prime} .\end{cases}
$$

Let $\psi \in C(\bar{\Omega})$ be such that

$$
\Omega_{+}^{\psi}:=\{x \in \Omega: \psi(x)>0\} \neq \emptyset
$$


Then, we introduce the condition

$$
\Omega_{+}^{\psi} \text { consists of a finite number of connected components of } \Omega . \quad\left(H_{\psi}\right)
$$

We shall assume this condition for $\psi=a$ and $\psi=-a$.

Motivated by the model case (1.1), we assume that

$$
\lim _{s \rightarrow 0^{+}} s^{1-q} f^{\prime}(s)=: f_{0} \in(0, \infty) \text { for some } q \in(0,1) .
$$

We will see that under this condition $f$ behaves like $\frac{f_{0}}{q} s^{q}$ when $s \rightarrow 0^{+}$, and satisfies the slope condition, see Remark 2.1. In addition, the following strong concavity (respect. convexity) condition on $f$ (respect. $g$ ) shall be used:

$$
\begin{aligned}
& \left(\frac{f(s)}{s^{q}}\right)^{\prime} \leq 0 \text { for } s>0, \\
& \left(\frac{g(s)}{s}\right)^{\prime}>0 \text { for } s>0,
\end{aligned}
$$

where $q \in(0,1)$ is given by (1.9). We introduce now the Gidas-Spruck condition [13, Theorem 1.1], which is stronger than (1.5):

$$
0<\lim _{s \rightarrow \infty} \frac{g(s)}{s^{p}}<\infty \quad \text { for some } p>1 \text {, where } p<\frac{N+2}{N-2} \text { if } N>2 .
$$

Finally, we shall use the condition $\left(\mathcal{H}_{b}\right)$, which will be precisely stated in Remark 2.3 and goes back to Amann and López-Gómez [2].

For our second purpose, we focus on the case $f(s)=s^{q}, q \in(0,1)$. In association with the sublinear problem

$$
\begin{cases}-\Delta u=a(x) u^{q} & \text { in } \Omega, \\ \mathcal{B} u=0 & \text { on } \partial \Omega,\end{cases}
$$

we introduce the set

$\mathcal{A}_{\mathcal{B}}^{a}:=\{q \in(0,1): u \gg 0$ for any nontrivial nonnegative solution $u$ of $(1.13)\}$.

We know [15. Corollary 1.5 and Theorem 1.9] that under $\left(H_{\psi}\right)$ with $\psi=a$, there exists $q_{a} \in[0,1)$ such that $\mathcal{A}_{\mathcal{B}}^{a}=\left(q_{a}, 1\right)$, assuming additionally $\int_{\Omega} a<0$ if $\mathcal{B} u=\frac{\partial u}{\partial \mathbf{n}}$. Let us point out that the condition $\int_{\Omega} a<0$ is necessary and sufficient for the existence of a positive solution of (1.13) with $\mathcal{B} u=\frac{\partial u}{\partial \mathbf{n}}$, for some $q \in(0,1)$, see [16, Corollary 1.3].

We are now in position to state our main results. First, we deal with the Dirichlet problem.

Theorem 1.1. Under $\mathcal{B} u=u$, we assume (1.3), (1.4), (1.7), (1.9), (1.12), and $\left(H_{\psi}\right)$ with $\psi= \pm a$. In addition, suppose either

(a) $b>0$ on $\bar{\Omega}$, and $p<\frac{N+2}{N-2}$ if $N>2$, or

(b) (1.10), (1.11), and $\left(\mathcal{H}_{b}\right)$.

Then, the following two assertions hold: 
(i) $\left(P_{\mathcal{B}}\right)$ admits a loop type subcontinuum $\mathcal{C}_{0}$ (i.e., a nonempty, closed and connected subset in $\mathbb{R} \times C_{0}^{1}(\bar{\Omega})$ ) of nonnegative solutions which satisfies (1.6). Moreover, we have the following properties, see Figure 1(i):

(1) $\left(0, u_{0}\right) \in \mathcal{C}_{0}$ for some positive solution $u_{0}$ of $\left(P_{\mathcal{B}}\right)$ with $\lambda=0$.

(2) There exists $\delta>0$ such that $\mathcal{C}_{0}$ does not contain any positive solution $u$ of $\left(P_{\mathcal{B}}\right)$ with $\lambda=0$ satisfying $\|u\|_{C(\bar{\Omega})} \leq \delta$.

(3) $\mathcal{C}_{0}$ contains closed connected sets $\mathcal{C}_{0}^{ \pm}$such that $\{(0,0)\} \subsetneq \mathcal{C}_{0}^{ \pm}$, and if $(\lambda, u) \in \mathcal{C}_{0}^{ \pm} \backslash\{(0,0)\}$, then $\lambda \gtrless 0$, i.e., $\mathcal{C}_{0}$ bifurcates both subcritically and supercritically at $(0,0)$.

(ii) Let $f(s)=s^{q}, q \in(0,1)$. Assume that $b \geq 0$, and additionally that

$$
g(s) \geq 0 \quad \text { for } s>0
$$

when condition (a) holds. If $q \in \mathcal{A}_{\mathcal{B}}^{a} \cap \mathcal{A}_{\mathcal{B}}^{-a}$, then $u \gg 0$ for any $(\lambda, u) \in$ $\mathcal{C}_{0} \backslash\{(0,0)\}$. In particular, the component of nontrivial nonnegative solutions of $\left(P_{\mathcal{B}}\right)$ including $\mathcal{C}_{0} \backslash\{(0,0)\}$ is bounded.

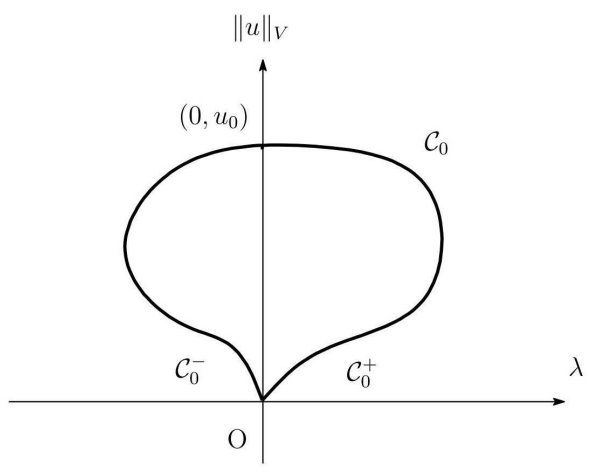

(i)

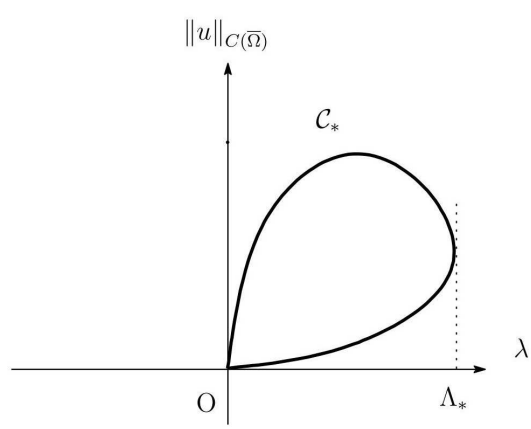

(ii)

Figure 1. The loop type subcontinua $\mathcal{C}_{0}$ and $\mathcal{C}_{*}$.

Next we consider the Neumann problem under the condition

$$
\int_{\Omega} b<0
$$

In this case, we shall obtain a loop type subcontinuum of nonnegative solutions with the same nature the Dirichlet case admits, as in Figure 1(i) (for the case $\int_{\Omega} b \geq 0$ we refer to Remark 1.3 below). To this end, we need the following decay and positivity condition for $g$, which is stronger than (1.3):

$$
\lim _{s \rightarrow 0^{+}} \frac{g(s)}{s^{\sigma}}=: g_{0} \in(0, \infty) \quad \text { for some } \sigma>1 \text {, where } \sigma<\frac{2 N}{N-2} \text { if } N>2 .
$$


Moreover, we are able to discuss the positivity of (nontrivial) nonnegative solutions for $\left(P_{\mathcal{B}}\right)$ with (1.1), assuming

$$
\left\{\begin{array}{l}
p<\frac{N+1}{N-1} \text { if } N>2 \\
a \in C^{\alpha}(\bar{\Omega}) \text { for some } \alpha \in(0,1) \\
\int_{\Omega} a<0 \\
b \equiv 1 .
\end{array}\right.
$$

It is known [26, Theorem 1] that if $\Omega_{+}^{a}$ is connected, then $\left(P_{\mathcal{B}}\right)$ possesses a loop type subcontinuum $\mathcal{C}_{*}$ in $\mathbb{R} \times C(\bar{\Omega})$ of nonnegative solutions which satisfies (1.6). Furthermore, we have the following properties, see Figure 1(ii):

(i) $\left\{\lambda:(\lambda, u) \in \mathcal{C}_{*} \backslash\{(0,0)\}\right\}=\left(0, \Lambda_{*}\right]$ for some $\Lambda_{*}>0$.

(ii) $\mathcal{C}_{*}$ possesses at least two nontrivial nonnegative solutions for $\lambda>0$ small enough.

Now, we state our main results for the Neumann problem, which are given in a similar way as in Theorem [1.1, and where condition (1.16) provides us with a loop type subcontinuum bifurcating both subcritically and supercritically at $(0,0)$.

Theorem 1.2. Under $\mathcal{B} u=\frac{\partial u}{\partial \mathbf{n}}$, assume (1.7), (1.9), (1.10), (1.11), (1.12), (1.17), $\left(H_{\psi}\right)$ with $\psi= \pm a$, and $\left(\mathcal{H}_{b}\right)$. Then, the following two assertions hold:

(i) If (1.16) holds, then $\left(P_{\mathcal{B}}\right)$ admits a loop type subcontinuum $\mathcal{C}_{*}$ in $\mathbb{R} \times C^{1}(\bar{\Omega})$ of nonnegative solutions for which the same assertions in Theorem 1.1(i) hold true, see Figure $1(i)$.

(ii) Assume (1.1), (1.18) and the condition that $\Omega_{+}^{a}$ is connected. Let $\mathcal{C}_{*}$ be the loop type subcontinuum stated above. If $q \in \mathcal{A}_{\mathcal{B}}^{a}$, then the same conclusion in Theorem 1.1( (ii) holds with $\mathcal{C}_{0}$ replaced by $\mathcal{C}_{*}$.

Remark 1.3. When $\mathcal{B} u=\frac{\partial u}{\partial \mathbf{n}}$ and $\int_{\Omega} b(x) \geq 0$, the existence of a loop type subcontinuum of nonnegative solutions of $\left(P_{\mathcal{B}}\right)$ has been established in the particular case $f(s)=s^{q}$ and $g(s)=s^{p}$ with $0<q<1<p$ (see [26], and as a particular case, see also (1.18)). In this case, although the loop type subcontinuum $\mathcal{C}_{0}$ satisfies (1.6), $\mathcal{C}_{0} \backslash\{(0,0)\}$ appears in $\lambda>0$. This means that $\mathcal{C}_{0}$ never meets the vertical line $\{(0, u): 0 \not \equiv u \geq 0\}$, see [24, Lemma 6.8(1)]. Thus, the approach used in the proof of Theorem 1.2(i) does not work for excluding the possibility that $\mathcal{C}_{0}=\{(0,0)\}$, see the argument in Subsection 5.1. Let us mention that in [26] the authors used a suitable rescaling technique (which strongly relies on the homogeneity of $f(s)=s^{q}$ and $\left.g(s)=s^{p}\right)$ to exclude this possibility.

Remark 1.4. Theorems 1.1 and 1.2 can be extended to the case $a, b \in L^{\infty}(\Omega)$ except assertion (ii) in Theorem 1.2 This can be done if we formulate $\left(H_{\psi}\right)$ for $\psi \in L^{\infty}(\Omega)$ such that $\psi \neq \equiv 0$, letting $\Omega_{+}^{\psi}$ be the largest open subset of $\Omega$ in which $\psi>0$ a.e., and assuming additionally

$$
\left\{\begin{array}{l}
\psi \text { is bounded away from zero on compact subsets of } \Omega_{i}, \\
\text { for } i=1, \ldots, l \text {, and }\left|\left(\operatorname{supp} \psi^{+}\right) \backslash \Omega_{+}^{\psi}\right|=0
\end{array}\right.
$$




\section{Preliminaries And Examples}

We start this section with some remarks concerning some of our assumptions.

Remark 2.1. Condition (1.9) implies:

(i) by the L'Hospital rule,

$$
\lim _{s \rightarrow 0^{+}} \frac{f(s)}{s^{q}}=\frac{f_{0}}{q}>0 .
$$

In particular, since $f \in C^{1}((0, \infty))$ and $f(0)=0$, we can show that $f \in$ $C^{\alpha}\left(\left[0, s_{0}\right]\right)$ for $\alpha \in(0, q]$ and $s_{0}>0$.

(ii) $f$ satisfies the slope condition, that is, for any $s_{0}>0$, there exists $M_{0}>0$ such that

$$
\frac{f(s)-f(t)}{s-t}>-M_{0} \quad \text { for } 0 \leq t<s \leq s_{0} .
$$

However, even under (1.9),$a(x) f(\cdot)$ does not satisfy the slope condition for $x \in \Omega$ where $a(x)<0$, since $\lim _{s \rightarrow 0^{+}} f^{\prime}(s)=\infty$.

\section{Remark 2.2.}

(i) Since $f(s)>0$ for $s>0$, we note that if (1.10) holds, then $f$ is concave for $s>0$, i.e.

$$
\left(\frac{f(s)}{s}\right)^{\prime}<0 \text { for } s>0 .
$$

(ii) It is easy to check that (1.10) is stronger than (1.4). This is a consequence of the fact that (1.10) yields

$$
0 \leq \lim _{s \rightarrow \infty} \frac{f(s)}{s^{q}}<\infty .
$$

(iii) Let us also note that (1.3) and (1.11) imply that $g(s)>0$ for $s>0$. Indeed, assume first $g\left(s_{0}\right)<0$ for some $s_{0}>0$, and set $\varepsilon_{0}:=-g\left(s_{0}\right) / s_{0}>0$. From (1.3), we infer that for some $s_{1} \in\left(0, s_{0}\right)$,

$$
\frac{g\left(s_{0}\right)}{s_{0}}=-\varepsilon_{0}<\frac{g\left(s_{1}\right)}{s_{1}},
$$

which contradicts (1.11). Hence $g(s) \geq 0$ for all $s>0$. Next, assume that $g\left(s_{0}\right)=0$ for some $s_{0}>0$. By (1.11), it follows that $g(s) \not \equiv 0$ for $s \in\left(0, s_{0}\right)$. This implies that $g\left(s_{1}\right)>0$ for some $s_{1} \in\left(0, s_{0}\right)$. It follows that

$$
\frac{g\left(s_{1}\right)}{s_{1}}>0=\frac{g\left(s_{0}\right)}{s_{0}},
$$

which contradicts (1.11) again, as desired. 
Remark 2.3. We describe here the explicit formula for the growth condition $\mathcal{H}_{b}$ of $b^{+}$in a neighborhood of $\partial \Omega_{+}^{b}$ used in Theorems 1.1 and 1.2 which originates from Amann and López-Gómez [2, Theorem 4.3]:

$\Omega_{+}^{b}$ is a subdomain of $\Omega$ with smooth boundary $\partial \Omega_{+}^{b}$ and either

- $\overline{\Omega_{+}^{b}} \subset \Omega$, and $b<0$ in $D_{b}:=\Omega \backslash \overline{\Omega_{+}^{b}}$, or

- $\Omega_{+}^{b} \supset\{x \in \Omega: d(x, \partial \Omega)<\sigma\}$ for some $\sigma>0$, and $b<0$ in $D_{b}$.

In addition, $D_{b}$ is a subdomain of $\Omega$ with smooth boundary, and

there exist $\gamma>0$ and a function $\beta$ defined in a tubular neighborhood

$U:=\left\{x \in \Omega_{+}^{b}: d\left(x, \partial \Omega_{+}^{b}\right)<\sigma\right\}$ of $\partial \Omega_{+}^{b}$ in $\Omega_{+}^{b}$, which is continuous,

positive and bounded away from zero, and satisfies

$b^{+}(\cdot)=\beta(\cdot) d\left(\cdot, \partial \Omega_{+}^{b}\right)^{\gamma}$ in $U$ and $1<p<\min \left(\frac{N+2}{N-2}, \frac{N+1+\gamma}{N-1}\right)$ if $N>2$.

We conclude this section showing some examples of functions satisfying the previous conditions. We start with the following lemma, which characterizes the functions satisfying (1.9) and (1.10).

Lemma 2.4. Let $f \in C([0, \infty)) \cap C^{1}((0, \infty))$ with $f(0)=0$ and $f(s)>0$ for $s>0$. Then, the following two conditions are equivalent:

(i) (1.9) and (1.10) hold.

(ii) $f(s)=s^{q} h(s)$ for some $q \in(0,1)$ and $h \in C([0, \infty)) \cap C^{1}((0, \infty))$ such that $h$ is nonincreasing, $h(s)>0$ for $s \geq 0$ and $\lim _{s \rightarrow 0^{+}} s h^{\prime}(s)=0$.

Proof. It is easy to see that $f$ as in condition (ii) fulfills (1.9) and (1.10). Conversely, if $f$ satisfies the aforementioned conditions, defining $h(s):=s^{-q} f(s)$ for $s>0$ and $h(0):=\lim _{s \rightarrow 0^{+}} h(s)$, it is also easy to check that $h$ has the desired properties.

As particular cases, we mention $h(s)=\frac{1}{1+s^{r}}(r \geq 0)$ and $h(s)=e^{-s}$.

We note that oscillatory cases are out of our scope. For instance, consider $h(s)=\sin \left(\frac{1}{s}\right)+2$. If we put $f(s):=s^{q} h(s)$ for $s>0$, and $f(0):=0$, then $f \in C([0, \infty)) \cap C^{1}((0, \infty))$ with $f(0)=0$ and $f(s)>0$ for $s>0$. Moreover, $f$ fulfills (1.2) and (1.4), but (2.1), (2.2) and (1.10) do not hold.

We now exhibit examples of $g$ satisfying (1.11), (1.12), and (1.17).

(a) We set

$$
g(s):=s^{p} h(s), \quad \text { with } \quad 1<p<\frac{N+2}{N-2} \quad \text { if } \quad N>2,
$$

where $h \in C^{1}([0, \infty))$ is nondecreasing, bounded, and satisfies one of the following conditions:

(i) $0=h(0)<h^{\prime}(0)$.

(ii) $0<h(0)$. 
Note that (1.17) holds if we choose $\sigma:=p+1$ in (i), and $\sigma:=p$ in (ii). An example for (i) is $h(s)=1-e^{-s}$, while $h(s)=\arctan (s+1)$ is included in (ii). Another example is given by $h(s)=\frac{s^{r}}{1+s^{r}}$, with $r=0$ or $r=1$. The case $r=1$ satisfies (i), whereas $r=0$ satisfies (ii). More generally, the function

$g(s)=\frac{s^{p+r}}{1+s^{r}}\left(0 \leq r<\frac{2 N}{N-2}-p\right)$ fulfills (1.11), (1.12), and (1.17). Indeed, we can take $\sigma:=p+r$ for (1.17).

(b) Let $k \geq 1$ and $1<p<\frac{N+2}{N-2}$ if $N>2$. We put

$$
g(s):=s^{p} \frac{k+s}{1+s}
$$

Then, $g$ satisfies (1.12) and (1.17). It is also clear that (1.11) holds when $k=1$. Meanwhile, when $k>1$, it satisfies (1.11) if additionally

$$
p>p_{1}(k), \text { where } p_{1}(k):=\frac{2 \sqrt{k}}{\sqrt{k}+1} .
$$

We note that $p_{1}(k)$ is increasing for $k>1$, and $p_{1}(k) \searrow 1$ as $k \rightarrow 1^{+}$, whereas $p_{1}(k) \nearrow 2$ as $k \rightarrow \infty$. Let us finally observe that case (b) is not included in any of the possibilities considered in case (a). Indeed, $h(s)=\frac{k+s}{1+s}$ is decreasing for $s \geq 0$.

\section{RegularizATION SCHEMES AND TRANSVERSALITY CONDITIONS}

Let us now explain our approach to study bifurcation of nontrivial nonnegative solutions for $\left(P_{\mathcal{B}}\right)$ from $(\lambda, 0)$. From (1.2), we see that $f$ is not differentiable at $s=0$, so that we can not directly apply the usual bifurcation theory from simple eigenvalues to $\left(P_{\mathcal{B}}\right)$. To overcome this difficulty, we proceed as in [24, 26, 'regularizing' $\left(P_{\mathcal{B}}\right)$ at $u=0$, using $\varepsilon>0$. We refer to [20, Section 5] for a similar approach introducing a new parameter for a different regular problem.

We extend $g$ to $\mathbb{R}$ as a $C^{1}$ function and set $F: \mathbb{R} \rightarrow \mathbb{R}$ by

$$
F(s):= \begin{cases}s^{1-q} f(s), & s \geq 0 \\ \frac{f_{0}}{q} s, & s<0 .\end{cases}
$$

For $\varepsilon>0$ we shall study the auxiliary problem

$\left(P_{\mathcal{B}, \varepsilon}\right) \quad \begin{cases}-\Delta u=\lambda a(x)(u+\varepsilon)^{q-1} F(u)+b(x) g(u) & \text { in } \Omega, \\ \mathcal{B} u=0 & \text { on } \partial \Omega .\end{cases}$

Note that (1.9) and (2.1) imply that $F \in C^{1}(\mathbb{R}), F(0)=0$ and $F^{\prime}(0)=\frac{f_{0}}{q}$, so that

$$
s \mapsto(s+\varepsilon)^{q-1} F(s) \in C^{1}((-\varepsilon, \infty)) .
$$

Observe also that $\left(P_{\mathcal{B}, 0}\right)$ corresponds to $\left(P_{\mathcal{B}}\right)$, as far as nonnegative solutions are concerned.

Let us set

$$
h_{\lambda, \varepsilon}(x, s):=\lambda a(x)(s+\varepsilon)^{q-1} F(s)+b(x) g(s) .
$$


From (3.2) we see that $h(x, \cdot)$ satisfies the slope condition. Consequently, given a nontrivial nonnegative solution $u$ of $\left(P_{\mathcal{B}, \varepsilon}\right)$, we can choose $M>0$ such that $(-\Delta+M) u \geq 0$ and $\not \equiv 0$ in $\Omega$. Thus, by the strong maximum principle and Hopf's lemma, we deduce $u \gg 0$, see [14, [19, Theorem 7.10].

We shall then consider the linearized eigenvalue problem at $u=0$ for the regular problem $\left(P_{\mathcal{B}, \varepsilon}\right)$ :

$$
\begin{cases}-\Delta \phi=\lambda a(x) \frac{f_{0}}{q} \varepsilon^{q-1} \phi & \text { in } \Omega, \\ \mathcal{B} \phi=0 & \text { on } \partial \Omega .\end{cases}
$$

Since $a$ changes sign, (3.3) has exactly two principal eigenvalues $\lambda_{1, \varepsilon}^{-}<0<\lambda_{1, \varepsilon}^{+}$ (respect. $\lambda_{1, \varepsilon}^{-}=0<\lambda_{1, \varepsilon}^{+}$) if

$$
\mathcal{B} u=u \quad\left(\text { respect. } \mathcal{B} u=\frac{\partial u}{\partial \mathbf{n}} \text { and } \int_{\Omega} a<0\right),
$$

which are both simple, and furthermore, $\left(\lambda_{1, \varepsilon}^{ \pm}, 0\right)$ satisfy the Crandall-Rabinowitz transversality condition, see [19, Theorem 9.4].

Thanks to the simplicity and transversality condition, the local bifurcation theory [9, Theorem 1.7] ensures the existence and uniqueness of positive solutions of $\left(P_{\mathcal{B}, \varepsilon}\right)$ bifurcating at $\left(\lambda_{1, \varepsilon}^{ \pm}, 0\right)$. Moreover, the unilateral global bifurcation theory [18, Theorem 6.4.3] (see also [23, Theorem 1.27]) ensures that $\left(P_{\mathcal{B}, \varepsilon}\right)$ possesses two components $\mathcal{C}_{\varepsilon}^{ \pm}=\{(\lambda, u)\}$ (i.e., maximal, nonempty, closed and connected subsets in $\mathbb{R} \times V)$ of nonnegative solutions emanating from $\left(\lambda_{1, \varepsilon}^{ \pm}, 0\right)$, respectively (see Remark 3.2). In addition, $\mathcal{C}_{\varepsilon}^{+} \backslash\left\{\left(\lambda_{1, \varepsilon}^{ \pm}, 0\right)\right\}$ and $\mathcal{C}_{\varepsilon}^{-} \backslash\left\{\left(\lambda_{1, \varepsilon}^{ \pm}, 0\right)\right\}$ consist of positive solutions. This is due to elliptic regularity and the fact (see [1, Proposition 18.1]) that $\left(P_{\mathcal{B}, \varepsilon}\right)$ has no bifurcating positive solutions from $(\lambda, 0)$ at any $\lambda \neq \lambda_{1, \varepsilon}^{ \pm}$.

Under some additional growth condition on $g$, we shall verify that $\mathcal{C}_{\varepsilon}^{ \pm}$are bounded in $\mathbb{R} \times V$ uniformly in $\varepsilon \in(0,1]$, so that $\mathcal{C}_{\varepsilon}^{-}=\mathcal{C}_{\varepsilon}^{+}\left(:=\mathcal{C}_{\varepsilon}\right)$ (i.e. $\mathcal{C}_{\varepsilon}$ is a mushroom). By simple computations, it can be shown easily that

$$
\lambda_{1, \varepsilon}^{ \pm} \rightarrow 0 \text { as } \varepsilon \rightarrow 0^{+},
$$

so that, passing to the limit as $\varepsilon \rightarrow 0^{+}$, we shall observe by Whyburn's topological argument [28, (9.12) Theorem] that

$$
\mathcal{C}_{0}:=\limsup _{\varepsilon \rightarrow 0^{+}} \mathcal{C}_{\varepsilon}
$$

is a loop type subcontinuum which consists of nonnegative solutions of $\left(P_{\mathcal{B}}\right)$ and satisfies (1.6).

Remark 3.1. The critical case

$$
\mathcal{B} u=\frac{\partial u}{\partial \mathbf{n}} \text { and } \int_{\Omega} a=0
$$

can be handled in a similar way. In this case, we replace $a$ by $a-\varepsilon$ for $\varepsilon>0$ small in $\left(P_{\mathcal{B}, \varepsilon}\right)$. Then, the above argument remains valid, since we can determine the asymptotic behavior (3.4) (see [24, Lemma 6.6] for the proof). In addition, we can reduce the case $\int_{\Omega} a>0$ to the case $\int_{\Omega} a<0$ under $\mathcal{B} u=\frac{\partial u}{\partial \mathbf{n}}$. Indeed, we only 
have to notice the symmetry property $\lambda a(x)=(-\lambda)(-a(x))$. The situation may be illustrated by Figures 2 (i) and (ii).

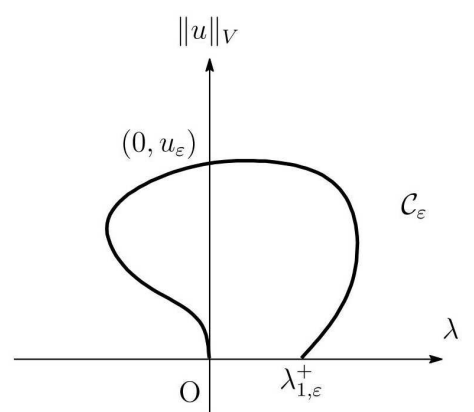

(i)

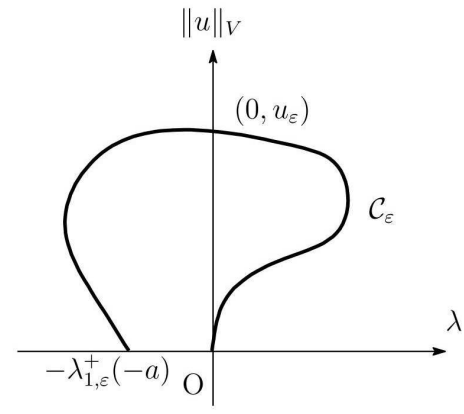

(ii)

Figure 2. The bounded component $\mathcal{C}_{\varepsilon}$ for $\left(P_{\mathcal{B}, \varepsilon}\right)$ with $\mathcal{B} u=\frac{\partial u}{\partial \mathbf{n}}$.

(i) Case $\int_{\Omega} a<0$. (ii) Case $\int_{\Omega} a>0$.

Remark 3.2. We shall show that the transversality condition allows us to apply the unilateral global bifurcation result [18, Theorem 6.4.3] to $\left(P_{\mathcal{B}, \varepsilon}\right)$ at $\left(\lambda_{1, \varepsilon}^{ \pm}, 0\right)$. To this end, we reduce $\left(P_{\mathcal{B}, \varepsilon}\right)$ to an operator equation in $C(\bar{\Omega})$. Given $\xi \in L^{r}(\Omega)$, $r>N$, let $u \in W_{\mathcal{B}}^{2, r}(\Omega)$ be the unique solution of

$$
\begin{cases}(-\Delta+1) u=\xi(x) & \text { in } \Omega \\ \mathcal{B} u=0 & \text { on } \partial \Omega\end{cases}
$$

where

$$
W_{\mathcal{B}}^{2, r}(\Omega):= \begin{cases}\left\{u \in W^{2, r}(\Omega): u=0 \text { on } \partial \Omega\right\} & \text { if } \mathcal{B} u=u \\ W^{2, r}(\Omega) & \text { if } \mathcal{B} u=\frac{\partial u}{\partial \mathbf{n}}\end{cases}
$$

We introduce the solution operator $\mathcal{S}: L^{r}(\Omega) \rightarrow W_{\mathcal{B}}^{2, r}(\Omega)$ associated with (3.6), implying that $\mathcal{S}(\xi)=u$, which is bijective and homeomorphic. It follows that $\mathcal{S}: L^{\infty}(\Omega) \rightarrow W_{\mathcal{B}}^{2, r}(\Omega)$ is continuous, and moreover, $\mathcal{S}: L^{\infty}(\Omega) \rightarrow V$ is compact, since so is the embedding $W_{\mathcal{B}}^{2, r}(\Omega) \subset V$. Thus, $\left(P_{\mathcal{B}, \varepsilon}\right)$ is reduced to

$$
\mathcal{F}(\lambda, u):=u-\mathcal{S}\left[\left(\lambda a \frac{f_{0}}{q} \varepsilon^{q-1}+1\right) u+h_{\lambda}(x, u)\right]=0 \quad \text { in } \quad C(\bar{\Omega}),
$$

where

$$
h_{\lambda}(x, s):=\lambda a(x)\left\{(s+\varepsilon)^{q-1} F(s)-\frac{f_{0}}{q} \varepsilon^{q-1} s\right\}+b(x) g(s) .
$$

Given $u \in C(\bar{\Omega})$, let

$$
\mathcal{A}_{\lambda} u:=\mathcal{S}\left[\left(\lambda a \frac{f_{0}}{q} \varepsilon^{q-1}+1\right) u\right]
$$

and let

$$
\operatorname{Ind}\left(0, \mathcal{A}_{\lambda}\right):=\operatorname{deg}\left(1-\mathcal{A}_{\lambda}, B_{R}\right)
$$


be the fixed point index of $\mathcal{A}_{\lambda}$ at the origin for $\lambda \neq \lambda_{1, \varepsilon}^{ \pm}$but close to $\lambda_{1, \varepsilon}^{ \pm}$, where $B_{R}$ is the ball with radius $R>0$ and centered at the origin. According to [18, Section 4.2, Theorem 5.6.2], the transversality condition at $\left(\lambda_{1, \varepsilon}^{ \pm}, 0\right)$ implies that $\operatorname{Ind}\left(0, \mathcal{A}_{\lambda}\right)$ changes sign as $\lambda$ crosses $\lambda_{1, \varepsilon}^{ \pm}$. Thus, [18, Theorem 6.4.3] applies, so that equation (3.7) (and so, $\left(P_{\mathcal{B}, \varepsilon}\right)$ ) possesses two components $\mathcal{C}_{\varepsilon}^{ \pm}=\{(\lambda, u)\}$ in $\mathbb{R} \times C(\bar{\Omega})$ of nonnegative solutions $u$ emanating from $\left(\lambda_{1, \varepsilon}^{ \pm}, 0\right)$, respectively. Finally, we can verify that $\mathcal{C}_{\varepsilon}^{ \pm}$are also components in $\mathbb{R} \times V$, by elliptic regularity.

In the forthcoming sections, we will characterize the limiting behavior of $\mathcal{C}_{\varepsilon}$ as $\varepsilon \rightarrow 0^{+}$under the conditions on $a, b, f$ and $g$ stated in Theorems 1.1 and 1.2 .

\section{A PRIORI BOUNDS}

In this section, we establish an a priori bound for positive solutions of the regularized problem $\left(P_{\mathcal{B}, \varepsilon}\right)$ in $\mathbb{R} \times V$ (Corollary 4.6).

We start with an a priori bound on $\lambda \in \mathbb{R}$, uniformly in $\varepsilon \in[0,1]$.

Proposition 4.1. Assume (1.3), (1.5), (1.9) and (1.7). Then, there exist $\bar{\lambda}, \bar{\varepsilon}>0$ such that if $0 \leq \varepsilon \leq \bar{\varepsilon}$, then $\left(P_{\mathcal{B}, \varepsilon}\right)$ has no positive solution for $|\lambda| \geq \bar{\lambda}$.

Proof. Let us suppose first that we are not in the case (3.5). Let $0 \leq \varepsilon \leq 1$, and assume that $\left(P_{\mathcal{B}, \varepsilon}\right)$ has a positive solution $u$ for some $\lambda>0$. Let $B$ be given by (1.7), $\lambda_{B}>0$ be the first eigenvalue of the problem

$$
\begin{cases}-\Delta \phi=\lambda \phi & \text { in } B, \\ \phi=0 & \text { on } \partial B,\end{cases}
$$

and $\phi_{B} \in C^{2}(\bar{B})$ be a positive eigenfunction associated to $\lambda_{B}$. We extend $\phi_{B}$ to $\bar{\Omega}$ by setting $\phi_{B}=0$ in $\bar{\Omega} \backslash \bar{B}$, so that $\phi_{B} \in H_{0}^{1}(\Omega)$. Since $u>0$ on $\bar{B}$ and $\frac{\partial \phi_{B}}{\partial \nu}<0$ on $\partial B$, the divergence theorem yields that

$$
\lambda_{B} \int_{B} \phi_{B} u=\int_{B}-\Delta \phi_{B} u=\int_{B} \nabla \phi_{B} \nabla u-\int_{\partial B} \frac{\partial \phi_{B}}{\partial \nu} u>\int_{B} \nabla \phi_{B} \nabla u
$$

where $\nu$ is the outward unit normal to $\partial B$. On the other hand, we see that

$$
\int_{B} \nabla u \nabla \phi_{B}=\int_{B} b(x) g(u) \phi_{B}+\lambda \int_{B} a(x)(u+\varepsilon)^{q-1} F(u) \phi_{B},
$$

where $q$ is given by (1.9). It follows that

$$
\int_{B} u^{q} \phi_{B}\left\{b(x) \frac{g(u)}{u^{q}}+\lambda a(x)\left(\frac{u}{u+\varepsilon}\right)^{1-q} \frac{F(u)}{u}-\lambda_{B} u^{1-q}\right\}<0 .
$$

Now, for $(x, s) \in B \times(0, \infty)$, we set

$$
h(x, s):=b(x) \frac{g(s)}{s^{q}}+\lambda a(x)\left(\frac{s}{s+\varepsilon}\right)^{1-q} \frac{F(s)}{s}-\lambda_{B} s^{1-q} .
$$

By (1.5), there exists $s_{0}>0$ such that

$$
g(s) \geq \frac{\lambda_{B}}{b_{0}} s \quad \text { for } s>s_{0},
$$


where $b_{0}$ is from (1.7). Hence, since $f(s)>0$ for $s>0$ and $a(x) \geq 0$ a.e. in $B$, we deduce that if $\lambda>0, x \in B$ and $s>s_{0}$, then

$$
h(x, s) \geq b(x) \frac{g(s)}{s^{q}}-\lambda_{B} s^{1-q} \geq s^{1-q}\left(b_{0} \frac{g(s)}{s}-\lambda_{B}\right) \geq 0 .
$$

Let us now consider the case $0<s \leq s_{0}$. From (1.3), we can choose $K_{0}>0$ such that

$$
\left|\frac{g(s)}{s}\right| \leq K_{0} \quad \text { for } 0<s \leq s_{0} .
$$

Recalling (1.9) (or (2.1)), we set

$$
M_{1}:=\inf _{0<s \leq s_{0}} \frac{F(s)}{s}>0 .
$$

By putting $b_{\infty}:=\|b\|_{\infty}$, it follows that

$$
\begin{aligned}
h(x, s) & =s^{1-q}\left\{b(x) \frac{g(s)}{s}+\lambda a(x)\left(\frac{1}{s+\varepsilon}\right)^{1-q} \frac{F(s)}{s}-\lambda_{B}\right\} \\
& \geq s^{1-q}\left\{\lambda a_{0}\left(\frac{1}{s_{0}+1}\right)^{1-q} M_{1}-\left(\lambda_{B}+b_{\infty} K_{0}\right)\right\},
\end{aligned}
$$

so that $h(x, s) \geq 0$ for $x \in B$ and $0<s \leq s_{0}$ if

$$
\lambda \geq \bar{\lambda}:=\frac{\left(\lambda_{B}+b_{\infty} K_{0}\right)\left(s_{0}+1\right)^{1-q}}{a_{0} M_{1}} .
$$

Consequently, by (4.2), (4.3) and (4.4) we deduce that $\lambda<\bar{\lambda}$.

Next, let us verify the existence of a lower bound on $\lambda<0$ for the existence of a positive solution of $\left(P_{\mathcal{B}, \varepsilon}\right)$. In order to check this, we notice that if $\left(P_{\mathcal{B}, \varepsilon}\right)$ has a positive solution $u$ for some $\lambda<0$ and $\varepsilon \in[0,1]$, then

$$
-\Delta u=(-\lambda)(-a(x))(u+\varepsilon)^{q-1} F(u)+b(x) g(u) \text { in } \Omega .
$$

From (1.7), the desired conclusion follows arguing as above with $B$ now replaced by $B^{\prime}$.

It remains to consider case (3.5). However, it suffices to note that (1.7) implies that if $\varepsilon$ is small enough, then

$$
a_{\varepsilon}(x) \geq \frac{a_{0}}{2} \text { in } B, \quad \text { and }-a_{\varepsilon}(x) \geq a_{0}^{\prime} \text { in } B^{\prime} .
$$

The proof now follows in the same way as above.

Next, given a compact interval $I$, we establish an a priori upper bound for positive solutions of $\left(P_{\mathcal{B}, \varepsilon}\right)$ whenever $\lambda \in I$ and $\varepsilon \in[0,1]$. We start with the following preliminary lemma (see also [2, Theorem 4.1]).

Lemma 4.2. Assume (1.5), (1.9), (1.10), (1.11) and $\left(\mathcal{H}_{b}\right)$. Let $\Lambda>0$. Suppose there exists a constant $C_{1}>0$ such that $\|u\|_{C\left(\overline{\Omega_{+}^{b}}\right)} \leq C_{1}$ for all positive solutions $u$ of $\left(P_{\mathcal{B}, \varepsilon}\right)$ with $\lambda \in[-\Lambda, \Lambda]$ and $\varepsilon \in[0,1]$. Then, there exists $C_{2}>0$ such that $\|u\|_{C(\bar{\Omega})} \leq C_{2}$ for all positive solutions $u$ of $\left(P_{\mathcal{B}, \varepsilon}\right)$ with $\lambda \in[-\Lambda, \Lambda]$ and $\varepsilon \in[0,1]$. 
Proof. (i) First, we consider the Dirichlet case. We use a comparison principle for concave problems inspired by the one in [3, Lemma 3.3].

(1) Assume first that $\overline{\Omega_{+}^{b}} \subset \Omega$, and recall that $D_{b}$ is given by $\left(\mathcal{H}_{b}\right)$. Let $\lambda \in[0, \Lambda]$, and consider the problem

$$
\begin{cases}-\Delta v=\lambda a^{+}(x)(v+\varepsilon)^{q-1} F(v)-b^{-}(x) g(v) & \text { in } D_{b}, \\ v=C_{1} & \text { on } \partial \Omega_{+}^{b}, \\ v=0 & \text { on } \partial \Omega .\end{cases}
$$

Let $u$ be a positive solution of $\left(P_{\mathcal{B}, \varepsilon}\right)$, with $\lambda \in[0, \Lambda]$ and $\varepsilon \in[0,1]$. It follows that $u>0$ in $D_{b}$. Since $\lambda \geq 0, b=-b^{-}$in $D_{b}$, and $f(s)>0$ for $s>0$, it is easy to check that $u$ is a subsolution of (4.5).

Next, we construct a supersolution of (4.5). Consider the unique positive solution $w_{0}$ of the problem

$$
\begin{cases}-\Delta w=1 & \text { in } D_{b} \\ w=0 & \text { on } \partial \Omega_{+}^{b} \cup \partial \Omega .\end{cases}
$$

Set $\bar{w}:=C\left(w_{0}+1\right)$ for $C>0$. If $C \geq C_{1}$, then $\bar{w} \geq C_{1}$ on $\partial \Omega_{+}^{b}$, and $\bar{w} \geq 0$ on $\partial \Omega$. Moreover, we claim that if $C$ is sufficiently large, then

$$
-\Delta \bar{w} \geq \lambda a^{+}(x)(\bar{w}+\varepsilon)^{q-1} F(\bar{w})-b^{-}(x) g(\bar{w}) \quad \text { in } D_{b} .
$$

Indeed, let

$$
\delta:=\frac{1}{\Lambda\left\|a^{+}\right\|_{\infty}\left(\left\|w_{0}\right\|_{C\left(\overline{D_{b}}\right)}+1\right)}>0 .
$$

Then, from (1.10) and (1.5) there exists $s_{1}>0$ large enough such that if $s \geq s_{1}$, then

$$
\left.0 \leq g(s) \text {, and } f(s) \leq \delta s \text { (so that } F(s) \leq \delta s^{2-q}\right) .
$$

It follows that if $C \geq s_{1}$, then

$$
\begin{aligned}
& -\Delta \bar{w}-\left\{\lambda a^{+}(x)(\bar{w}+\varepsilon)^{q-1} F(\bar{w})-b^{-}(x) g(\bar{w})\right\} \\
& \geq C-\Lambda\left\|a^{+}\right\|_{\infty} \bar{w}^{q-1} \delta \bar{w}^{2-q} \\
& \geq C\left\{1-\delta \Lambda\left\|a^{+}\right\|_{\infty}\left(\left\|w_{0}\right\|_{C\left(\overline{D_{b}}\right)}+1\right)\right\}=0 \text { in } D_{b} .
\end{aligned}
$$

Thus the claim has been verified, and $\bar{w}$ is a supersolution of (4.5) if $C \geq \max \left(C_{1}, s_{1}\right)$. Note that $C$ can be chosen independently of $\lambda \in[0, \Lambda]$ and $\varepsilon \in[0,1]$.

Now, we see from (1.10) and (1.11) that the nonlinearity in (4.5) is concave, that is, if we set

$$
j(x, s):=\lambda a^{+}(x)(s+\varepsilon)^{q-1} F(s)-b^{-}(x) g(s), \quad x \in D_{b}, s>0,
$$

then

$$
\frac{\partial}{\partial s}\left(\frac{j(x, s)}{s}\right)<0, \quad \text { for } x \in D_{b}, s>0
$$


Indeed,

$$
\begin{aligned}
\frac{d}{d s}\left(\frac{(s+\varepsilon)^{q-1} F(s)}{s}\right) & =(q-1)(s+\varepsilon)^{q-2}\left(s^{-q} f(s)\right)+(s+\varepsilon)^{q-1} \frac{d}{d s}\left(s^{-q} f(s)\right) \\
& <0 .
\end{aligned}
$$

Reasoning as in [25, Proposition A.1] (whose argument is based on [3, Lemma 3.3]), we may deduce that $u \leq \bar{w}$ in $D_{b}$, so that

$$
u \leq C_{1}+\|\bar{w}\|_{C\left(\overline{D_{b}}\right)} \text { on } \bar{\Omega} .
$$

It remains to verify the case $-\Lambda \leq \lambda<0$. Note that any positive solution $u$ of $\left(P_{\mathcal{B}, \varepsilon}\right)$ with $\lambda \in[-\Lambda, 0)$ and $\varepsilon \in[0,1]$ satisfies

$$
-\Delta u=\mu(-a(x))(u+\varepsilon)^{q-1} F(u)+b(x) g(u) \text { in } D_{b},
$$

with $\mu:=-\lambda \in(0, \Lambda]$. Instead of (4.5), we consider the following concave problem:

$$
\begin{cases}-\Delta v=\mu a^{-}(x)(v+\varepsilon)^{q-1} F(v)-b^{-}(x) g(v) & \text { in } D_{b}, \\ v=C_{1} & \text { on } \partial \Omega_{+}^{b}, \\ v=0 & \text { on } \partial \Omega .\end{cases}
$$

Then, we see that $u$ is a subsolution of this problem. The remainder of the argument is identical to the one in the case $\lambda \in[0, \Lambda]$.

(2) Assume now that $\Omega_{+}^{b} \supset\{x \in \Omega: d(x, \partial \Omega)<\sigma\}$ for some $\sigma>0$. This case can be verified identically. Indeed, it suffices to replace (4.5) by the problem

$$
\begin{cases}-\Delta v=\lambda a^{+}(x)(v+\varepsilon)^{q-1} F(v)-b^{-}(x) g(v) & \text { in } D_{b}, \\ v=C_{1} & \text { on } \partial \Omega_{+}^{b} \cap \Omega .\end{cases}
$$

(ii) Lastly, we verify the Neumann case. Since $a_{\varepsilon} \leq a^{+}$and $-a_{\varepsilon} \leq a^{-}+1$, it suffices to replace (4.5) by

$$
\begin{cases}-\Delta v=\lambda a^{+}(x)(v+\varepsilon)^{q-1} F(v)-b^{-}(x) g(v) & \text { in } D_{b}, \\ v=C_{1} & \text { on } \partial \Omega_{+}^{b}, \\ \frac{\partial v}{\partial \mathbf{n}}=0 & \text { on } \partial \Omega,\end{cases}
$$

and (4.6) by

$$
\begin{cases}-\Delta v=\mu\left(a^{-}(x)+1\right)(v+\varepsilon)^{q-1} F(v)-b^{-}(x) g(v) & \text { in } D_{b}, \\ v=C_{1} & \text { on } \partial \Omega_{+}^{b}, \\ \frac{\partial v}{\partial \mathbf{n}}=0 & \text { on } \partial \Omega .\end{cases}
$$

The proof of Lemma 4.2 is now complete.

The following result is due to Gidas and Spruck [13, Theorem 1.1] and Amann and López-Gómez [2, Section 4] (see also López-Gómez, Molina-Meyer and Tellini 21, Section 6]).

Proposition 4.3. Assume (1.4), (1.9) and (1.12). In addition, suppose either 
(i) $b>0$ on $\bar{\Omega}$, and $p<\frac{N+2}{N-2}$ for the Dirichlet case $\left(p<\frac{N+1}{N-1}\right.$ for the Neumann case) if $N>2$, or

(ii) (1.10), (1.11), and $\left(\mathcal{H}_{b}\right)$ hold.

Then, given $\Lambda>0$, there exists $C>0$ such that if $0 \leq \varepsilon \leq 1$, then $u \leq C$ in $\Omega$ for all positive solutions $u$ of $\left(P_{\mathcal{B}, \varepsilon}\right)$ with $|\lambda| \leq \Lambda$.

\section{Remark 4.4.}

(i) The condition (i) for the Neumann case is based on $\left(\mathcal{H}_{b}\right)$ with $\gamma=0$ from Amann and López-Gómez [2, Section 4], not on Gidas and Spruck [13. Theorem 1.1].

(ii) Under the condition (ii), we can handle the case where $b>0$ in $\Omega$, and $b=0$ somewhere on $\partial \Omega$.

Proof. Case (i) under $\mathcal{B} u=u$ is verified in the same way as in the proof of 13 , Theorem 1.1].

Case (i) under $\mathcal{B} u=\frac{\partial u}{\partial \mathbf{n}}$ and case (ii) can be handled using the arguments in [24, Proposition 6.5] and [25, Proposition 4.2], which are based on [21, Section 6]. Indeed, based on (1.4), (1.12), and $\left(\mathcal{H}_{b}\right)$, we employ the argument developed by Amann and López-Gómez [2] to deduce that the hypothesis of Lemma 4.2 is fulfilled, so that the desired conclusion follows.

By $\|\cdot\|_{H}$ we denote the usual norm of $H=H_{0}^{1}(\Omega)$ when $\mathcal{B} u=u$ and of $H=H^{1}(\Omega)$ when $\mathcal{B} u=\frac{\partial u}{\partial \mathbf{n}}$. The next lemma follows easily by a bootstrap argument based on elliptic regularity and the Sobolev embedding theorem:

Lemma 4.5. Assume that there exist $C>0$ and $p \in\left(1, \frac{N+2}{N-2}\right)$ such that

$$
|g(s)| \leq C\left(1+s^{p}\right) \quad \text { for } s \geq 0 .
$$

Let $\Lambda>0$ and $u$ be a nonnegative solution of $\left(P_{\mathcal{B}, \varepsilon}\right)$ for $\lambda \in[-\Lambda, \Lambda]$ and $\varepsilon \in[0,1]$. Then, given $c_{1}>0$ there exists $c_{2}>0$ such that if $\|u\|_{H} \leq c_{1}$, then $\|u\|_{V} \leq c_{2}$.

With the aid of Lemma 4.5. Propositions 4.1 and 4.3 provide us with an a priori bound in $\mathbb{R} \times V$ for positive solutions of $\left(P_{\mathcal{B}, \varepsilon}\right)$ uniformly in $\varepsilon \in[0,1]$.

Corollary 4.6. Under the assumptions of Propositions 4.1 and 4.3, there exists $\bar{\varepsilon}, \bar{C}>0$ such that if $0 \leq \varepsilon \leq \bar{\varepsilon}$, then $|\lambda|+\|u\|_{V} \leq \bar{C}$ for all positive solutions $u$ of $\left(P_{\mathcal{B}, \varepsilon}\right)$.

Proof. Propositions 4.1 and 4.3 imply that there exist $\bar{\varepsilon}, C>0$ such that if $0 \leq \varepsilon \leq$ $\bar{\varepsilon}$, then $|\lambda|+\|u\|_{H} \leq C$ for all positive solutions $u$ of $\left(P_{\mathcal{B}, \varepsilon}\right)$, since $u$ satisfies

$$
\int_{\Omega}|\nabla u|^{2}=\lambda \int_{\Omega}\left\{a(x)(u+\varepsilon)^{q-1} F(u) u+b(x) g(u) u\right\} \leq C^{\prime}
$$

for some $C^{\prime}>0$. Lemma 4.5 provides then the desired conclusion.

We discuss now bifurcation of nontrivial nonnegative solutions for $\left(P_{\mathcal{B}}\right)$ from $(\lambda, 0)$ for $\lambda>0$. We prove the following preliminary lemma. 
Lemma 4.7. Assume (1.9) and $\left(H_{\psi}\right)$ with $\psi=a$. Let $\Lambda>0, \Omega^{\prime}$ be a nonempty connected open subset of $\Omega_{+}^{a}$, and $B \Subset \Omega^{\prime}$ be a ball. Then there exists $C_{\Lambda}>0$ such that if $\lambda \geq \Lambda$, then $\|u\|_{C(\bar{B})} \geq C_{\Lambda}$ for all nontrivial nonnegative solutions $u$ of $\left(P_{\mathcal{B}}\right)$ such that $u \neq \equiv 0$ in $\Omega^{\prime}$.

Proof. We use an argument based on sub and supersolutions. First of all, we remark that (1.9) implies (1.2) and (2.2), see Remark 2.1.

Let $\Lambda, \Omega^{\prime}$ and $B$ be as in the statement of this lemma, and let $\lambda \geq \Lambda$. Assume that $u$ is a nontrivial nonnegative solution of $\left(P_{\mathcal{B}}\right)$ such that $u \neq \equiv 0$ in $\Omega^{\prime}$. Set

$$
K_{1}:=\max _{0 \leq s \leq\|u\|_{C(\bar{\Omega})}}\left|g^{\prime}(s)\right| \geq 0 .
$$

Since $g(0)=0$, the mean value theorem provides some constant $\theta=\theta_{x} \in(0,1)$ such that $g(u)=g^{\prime}(\theta u) u$. Thus, using (4.7) we get that

$$
\begin{aligned}
& \left(-\Delta+b_{\infty} K_{1}+1\right) u \\
& =\lambda a(x) f(u)+\left(b_{\infty} K_{1}+1+b(x) g^{\prime}(\theta u)\right) u \geq u \geq 0 \text { and } \not \equiv 0 \text { in } \Omega^{\prime} .
\end{aligned}
$$

The strong maximum principle yields that $u>0$ in $\Omega^{\prime}$. Now, let $s_{0}>0$ be fixed. Then, the following two possibilities may occur: (i) $u \leq s_{0}$ on $\bar{B}$; (ii) $u>s_{0}$ somewhere on $\bar{B}$.

We consider case (i). We have $a_{0}:=\inf _{\bar{B}} a>0$, so that $u$ is a supersolution of the problem

$$
\begin{cases}-\Delta v+b_{\infty} K_{2} v=\lambda a_{0} f(v) & \text { in } B, \\ v=0 & \text { on } \partial B\end{cases}
$$

where

$$
K_{2}:=\max _{0 \leq s \leq s_{0}}\left|g^{\prime}(s)\right| \geq 0 .
$$

Indeed, $u \geq 0$ on $\partial B$. Moreover, since $f(s)>0$ for $s>0$, the mean value theorem shows that

$$
\begin{aligned}
-\Delta u+b_{\infty} K_{2} u-\lambda a_{0} f(u) & =\lambda\left(a(x)-a_{0}\right) f(u)+\left(b_{\infty} K_{2}+b(x) g^{\prime}(\theta u)\right) u \\
& \geq 0 \quad \text { in } B .
\end{aligned}
$$

To construct a subsolution of (4.8), we use the positive eigenfunction $\phi_{B}$ associated to the first eigenvalue $\lambda_{B}$ of (4.1) and such that $\left\|\phi_{B}\right\|_{C(\bar{B})}=1$. From (1.2), we find a constant $s_{1}>0$ small enough such that

$$
\frac{f(s)}{s} \geq \frac{\lambda_{B}+b_{\infty} K_{2}}{\Lambda a_{0}} \quad \text { for } 0<s \leq s_{1} .
$$

If $0<s \leq s_{1}$, then we observe that

$$
\begin{aligned}
-\Delta\left(s \phi_{B}\right)+b_{\infty} K_{2} s \phi_{B}-\lambda a_{0} f\left(s \phi_{B}\right) & \leq s \phi_{B}\left\{\lambda_{B}+b_{\infty} K_{2}-\Lambda a_{0} \frac{f\left(s \phi_{B}\right)}{s \phi_{B}}\right\} \\
& \leq 0 \quad \text { in } B .
\end{aligned}
$$


This implies that $s \phi_{B}$ is a subsolution of (4.8) whenever $0<s \leq s_{1}$. Now, since $u>0$ in $\Omega^{\prime}$, it follows that $u>0$ on $\bar{B}$. Furthermore, we assert that

$$
u \geq s_{1} \phi_{B} \quad \text { on } \bar{B} .
$$

By contradiction, we assume that $u \nsupseteq s_{1} \phi_{B}$. Then, since $u>0=s_{1} \phi_{B}$ on $\partial B$, we can choose $\sigma \in(0,1)$ such that $u-\sigma s_{1} \phi_{B} \geq 0$ on $\bar{B}$ and $u-\sigma s_{1} \phi_{B}=0$ somewhere in $B$. From (2.2), we fix $M_{0}>0$ such that

$$
\frac{f(s)-f(t)}{s-t}>-M_{0} \quad \text { for } 0 \leq t<s \leq s_{0} .
$$

Putting $M_{1}:=\lambda a_{0} M_{0}>0$, we see that the mapping

$$
s \longmapsto M_{1} s+\lambda a_{0} f(s)
$$

is nondecreasing for $0 \leq s \leq s_{0}$. Indeed, if $0 \leq t<s \leq s_{0}$, then

$$
\begin{aligned}
& M_{1} s+\lambda a_{0} f(s)-\left(M_{1} t+\lambda a_{0} f(t)\right) \\
& =\left(M_{1}+\lambda a_{0} \frac{f(s)-f(t)}{s-t}\right)(s-t) \\
& \geq\left(M_{1}-\lambda a_{0} M_{0}\right)(s-t)=0,
\end{aligned}
$$

as desired. Thus, using this monotonicity and having in mind that

$$
\left(-\Delta+b_{\infty} K_{2}\right) \sigma s_{1} \phi_{B} \leq \lambda a_{0} f\left(\sigma s_{1} \phi_{B}\right) \text { in } B \quad(\text { recall (4.9) }) \text {, }
$$

we deduce that

$$
\begin{aligned}
& \left(-\Delta+b_{\infty} K_{2}+M_{1}\right)\left(u-\sigma s_{1} \phi_{B}\right) \\
& \geq M_{1} u+\lambda a_{0} f(u)-\left(M_{1} \sigma s_{1} \phi_{B}+\lambda a_{0} f\left(\sigma s_{1} \phi_{B}\right)\right) \\
& \geq 0 \quad \text { in } B
\end{aligned}
$$

and $u-\sigma s_{1} \phi_{B}=u>0$ on $\partial B$. The strong maximum principle yields that $u-\sigma s_{1} \phi_{B}>0$ in $B$, a contradiction. Thus, we have verified (4.10). By taking into account case (ii), $C_{\Lambda}:=\min \left\{s_{0}, s_{1}\right\}$ is as desired.

By virtue of Lemma 4.7, there are no nontrivial nonnegative solutions of $\left(P_{\mathcal{B}}\right)$ bifurcating from $(\lambda, 0)$ for $\lambda>0$, and moreover, there exist no small positive solutions of $\left(P_{\mathcal{B}}\right)$ for $\lambda=0$. In view of this fact, although we shall observe that $\left(P_{\mathcal{B}}\right)$ possesses a bounded subcontinuum of nontrivial nonnegative solutions bifurcating at $(0,0)$, we infer that the bifurcation subcontinuum is of loop type.

Proposition 4.8. Assume (1.3). Then, the following assertions hold:

(i) Assume $\left(H_{\psi}\right)$ with $\psi=a$, and additionally (1.9) if $\mathcal{B} u=\frac{\partial u}{\partial \mathbf{n}}$. Then, given $\lambda_{0}>0$, there exist $\delta_{0}, c_{0}>0$ such that $\|u\|_{C(\bar{\Omega})} \geq c_{0}$ for all nontrivial nonnegative solutions $u$ of $\left(P_{\mathcal{B}}\right)$ for $\lambda \in\left(\lambda_{0}-\delta_{0}, \lambda_{0}+\delta_{0}\right)$.

(ii) Assume (1.16) and (1.17) if $\mathcal{B} u=\frac{\partial u}{\partial \mathbf{n}}$. Then, there exists $C>0$ such that $\|u\|_{C(\bar{\Omega})} \geq C$ for all positive solutions $u$ of $\left(P_{\mathcal{B}}\right)$ for $\lambda=0$. 
Proof. (i) We recall that (1.9) also implies (2.1), see Remark 2.1. First, we verify the Dirichlet case. By contradiction, we assume that $\lambda_{n} \rightarrow \lambda_{0}>0$, and $u_{n}$ are nontrivial nonnegative solutions of $\left(P_{\mathcal{B}}\right)$ with $\lambda=\lambda_{n}$ such that $\left\|u_{n}\right\|_{C(\bar{\Omega})} \rightarrow 0$. Then, we claim that, up to a subsequence, $\int_{\Omega} a(x) f\left(u_{n}\right) u_{n} \leq 0$. If not, then we may suppose that $\int_{\Omega} a(x) f\left(u_{n}\right) u_{n}>0$ for all $n$. It follows that $u_{n} \not \equiv 0$ in $\Omega_{+}^{a}$. Indeed, if $u_{n} \equiv 0$ in $\Omega_{+}^{a}$, then, using that $f(s)>0$ for $s>0$, we find that

$$
0<\int_{\Omega} a(x) f\left(u_{n}\right) u_{n} \leq \int_{\Omega_{+}^{a}} a(x) f\left(u_{n}\right) u_{n}=0,
$$

which is a contradiction. Employing $\left(H_{\psi}\right)$ with $\psi=a$, we may deduce that there exists a connected open subset $\Omega^{\prime} \subset \Omega_{+}^{a}$ such that $u_{n} \not \equiv 0$ in $\Omega^{\prime}$ for all $n \geq 1$. Let $B \Subset \Omega^{\prime}$ be a ball. We apply Lemma 4.7 with $\Lambda=\frac{\lambda_{0}}{2}$, to derive that $\left\|u_{n}\right\|_{C(\bar{B})} \geq c_{0}$ for some $c_{0}>0$ independent of $n$, which contradicts $\left\|u_{n}\right\|_{C(\bar{\Omega})} \rightarrow 0$. Thus, the claim follows.

Now, we observe from the definition of $u_{n}$ that

$$
\begin{aligned}
\left\|u_{n}\right\|_{H}^{2}:=\int_{\Omega}\left|\nabla u_{n}\right|^{2} & =\lambda_{n} \int_{\Omega} a(x) f\left(u_{n}\right) u_{n}+\int_{\Omega} b(x) g\left(u_{n}\right) u_{n} \\
& \leq \int_{\Omega} b_{\infty}\left|g\left(u_{n}\right)\right| u_{n} .
\end{aligned}
$$

Set $v_{n}:=u_{n} /\left\|u_{n}\right\|_{H}$, so that

$$
\left\|v_{n}\right\|_{H}^{2} \leq b_{\infty} \int_{\Omega} \frac{\left|g\left(u_{n}\right)\right| u_{n}}{\left\|u_{n}\right\|_{H}^{2}} .
$$

From (1.3), given $\varepsilon>0$ there exists $s_{\varepsilon}>0$ such that

$$
|g(s)| \leq \frac{\varepsilon}{b_{\infty}} s \quad \text { for } 0<s \leq s_{\varepsilon}
$$

Also, for $n$ large enough, we have that $\left\|u_{n}\right\|_{C(\bar{\Omega})} \leq s_{\varepsilon}$, so that

$$
b_{\infty} \int_{\Omega} \frac{\left|g\left(u_{n}\right)\right| u_{n}}{\left\|u_{n}\right\|_{H}^{2}} \leq \varepsilon \int_{\Omega} v_{n}^{2}
$$

From (4.11) and (4.12), we derive that $v_{n} \rightarrow 0$ in $H_{0}^{1}(\Omega)$, a contradiction.

Next, we verify the Neumann case. Assume to the contrary that $\lambda_{n} \rightarrow \lambda_{0}>$ 0 , and $u_{n}$ are nontrivial nonnegative solutions of $\left(P_{\mathcal{B}}\right)$ with $\lambda=\lambda_{n}$ such that $\left\|u_{n}\right\|_{C(\bar{\Omega})} \rightarrow 0$. We remark that $\left\|u_{n}\right\|_{H} \rightarrow 0$, since $u_{n}$ are nonnegative solutions of $\left(P_{\mathcal{B}}\right)$ with $\lambda=\lambda_{n}$. Arguing as in the proof for the Dirichlet case, we have that, up to a subsequence, $\int_{\Omega} a(x) f\left(u_{n}\right) u_{n} \leq 0$, and consequently, $\int_{\Omega}\left|\nabla u_{n}\right|^{2} \leq$ $b_{\infty} \int_{\Omega}\left|g\left(u_{n}\right)\right| u_{n}$.

Set $v_{n}:=u_{n} /\left\|u_{n}\right\|_{H}$, so that $\left\|v_{n}\right\|_{H}=1$. We may assume that there exists $v_{0} \in H^{1}(\Omega)$ such that $v_{n} \rightarrow v_{0}$ in $H^{1}(\Omega), v_{n} \rightarrow v_{0}$ a.e. in $\Omega$, and $v_{n} \rightarrow v_{0}$ in $L^{t}(\Omega)$ for $t<2^{*}$. By (1.3), for any $\varepsilon>0$ there exists $s_{0}>0$ such that

$$
|g(s)| \leq \frac{\varepsilon}{b_{\infty}} s \quad \text { for } 0 \leq s \leq s_{0}
$$


Thus, for $n$ large enough we have that $\left\|u_{n}\right\|_{C(\bar{\Omega})} \leq s_{0}$, so that

$$
\int_{\Omega}\left|\nabla v_{n}\right|^{2} \leq b_{\infty} \int_{\Omega} \frac{\left|g\left(u_{n}\right)\right|}{\left\|u_{n}\right\|_{H}} v_{n} \leq \varepsilon \int_{\Omega} v_{n}^{2} \leq \varepsilon
$$

This implies that $\int_{\Omega}\left|\nabla v_{n}\right|^{2} \rightarrow 0$, and it follows that $v_{n} \rightarrow v_{0}$, and $v_{0}$ is a positive constant.

Since $u_{n}$ is a nonnegative solution of $\left(P_{\mathcal{B}}\right)$ with $\lambda=\lambda_{n}$, we see that, for every $\phi \in H^{1}(\Omega)$,

$$
\left(\int_{\Omega} \nabla v_{n} \nabla \phi\right)\left\|u_{n}\right\|_{H}^{1-q}=\lambda_{n} \int_{\Omega} a(x) \frac{f\left(u_{n}\right)}{\left\|u_{n}\right\|_{H}^{q}} \phi+\int_{\Omega} b(x) \frac{g\left(u_{n}\right)}{\left\|u_{n}\right\|_{H}^{q}} \phi .
$$

Since $\left\|u_{n}\right\|_{C(\bar{\Omega})} \rightarrow 0$, (4.13) implies that $\left|g\left(u_{n}\right)\right| \leq u_{n}$ for $n$ large enough, so that

$$
\begin{aligned}
\left|\int_{\Omega} b(x) \frac{g\left(u_{n}\right)}{\left\|u_{n}\right\|_{H}^{q}} \phi\right| & \leq b_{\infty} \int_{\Omega} \frac{u_{n}}{\left\|u_{n}\right\|_{H}^{q}}|\phi| \\
& \leq b_{\infty}\left\|u_{n}\right\|_{H}^{1-q}\left(\int_{\Omega} v_{n}^{2}\right)^{\frac{1}{2}}\left(\int_{\Omega} \phi^{2}\right)^{\frac{1}{2}} \longrightarrow 0 .
\end{aligned}
$$

We use this inequality to deduce from (4.14) that, passing to the limit as $n \rightarrow \infty$,

$$
\int_{\Omega} a(x) \frac{f\left(u_{n}\right)}{\left\|u_{n}\right\|_{H}^{q}} \phi \longrightarrow 0 .
$$

On the other hand, since $f(0)=0$ we have that

$$
\int_{\Omega} a(x) \frac{f\left(u_{n}\right)}{\left\|u_{n}\right\|_{H}^{q}} \phi=\int_{v_{n}>0} a(x) \frac{f\left(\left\|u_{n}\right\|_{H} v_{n}\right)}{\left(\left\|u_{n}\right\|_{H} v_{n}\right)^{q}} v_{n}^{q} \phi .
$$

Thus, using (2.1) and the fact that $u_{n} \rightarrow 0$ in $H^{1}(\Omega), v_{n} \rightarrow v_{0}$ in $L^{t}(\Omega), v_{n} \rightarrow v_{0}$ a.e. in $\Omega$ and $v_{0}$ is a positive constant, the Lebesgue dominated convergence theorem yields that

Therefore

$$
\int_{\Omega} a(x) \frac{f\left(u_{n}\right)}{\left\|u_{n}\right\|_{H}^{q}} \phi \longrightarrow \frac{f_{0}}{q} v_{0}^{q} \int_{\Omega} a(x) \phi
$$

$$
\int_{\Omega} a(x) \phi=0 .
$$

Since $\phi \in H^{1}(\Omega)$ is arbitrary, we find that $a \equiv 0$, which is a contradiction.

(ii) In the Dirichlet case, we argue as in the proof of assertion (i) to prove assertion (ii), by taking $\lambda_{n}=\lambda_{0}=0$ therein.

Next, we verify the Neumann case. Assume to the contrary that there exist positive solutions $u_{n}$ of $\left(P_{\mathcal{B}}\right)$ for $\lambda=0$ such that $\left\|u_{n}\right\|_{C(\bar{\Omega})} \rightarrow 0$. Then, as in the proof of assertion (i), we may deduce from (1.3) that $v_{n}:=u_{n} /\left\|u_{n}\right\|_{H^{1}(\Omega)} \rightarrow v_{0}$ in $L^{t}(\Omega)$ for $t<2^{*}$, and $v_{0}$ is a positive constant. Since $u_{n}$ is a positive solution of $\left(P_{\mathcal{B}}\right)$ with $\lambda=0$, we obtain $\int_{\Omega} b(x) g\left(u_{n}\right)=0$. Recalling (1.17), we see that

$$
0=\int_{\Omega} b(x) \frac{g\left(u_{n}\right)}{\left\|u_{n}\right\|_{H^{1}(\Omega)}^{\sigma}} \longrightarrow g_{0} v_{0}^{\sigma} \int_{\Omega} b(x),
$$

so that $\int_{\Omega} b(x)=0$, which contradicts (1.16). 
The proof is now complete.

Assuming additionally $\left(H_{\psi}\right)$ with $\psi=-a$, we can extend Proposition 4.8(i) to $\lambda<0$, and in this case, bifurcation of nontrivial nonnegative solutions of $\left(P_{\mathcal{B}}\right)$ from $(\lambda, 0)$ can only occur at $(0,0)$.

Corollary 4.9. Under the assumptions of Proposition 4.8, assume in addition $\left(H_{\psi}\right)$ with $\psi=-a$. Then the conclusion of Proposition $4.8(i)$ holds for all $\lambda_{0} \neq 0$. In particular, given $\delta \in(0,1)$, the set of nontrivial nonnegative solutions of $\left(P_{\mathcal{B}}\right)$ is away from the set $\left\{(\lambda, 0) ; \delta \leq|\lambda| \leq \delta^{-1}\right\}$.

Proof. In view of Proposition 4.8, it remains to verify the case $\lambda<0$. Assume to the contrary that $\lambda_{n} \rightarrow \lambda_{0}<0$, and $u_{n}$ are nontrivial nonnegative solutions of $\left(P_{\mathcal{B}}\right)$ with $\lambda=\lambda_{n}$ such that $u_{n} \rightarrow 0$ in $C(\bar{\Omega})$. Then, we have that

$$
-\Delta u_{n}=\left(-\lambda_{n}\right)(-a(x)) f\left(u_{n}\right)+b(x) g\left(u_{n}\right) \quad \text { in } \Omega .
$$

By the same arguments used in Lemma 4.7 and Proposition 4.8, we get the desired conclusion.

\section{Proofs of Theorems 1.1 and 1.2}

If $X$ is a metric space and $E_{n} \subset X$, then we set

$$
\begin{aligned}
& \liminf _{n \rightarrow \infty} E_{n}:=\left\{x \in X: \lim _{n \rightarrow \infty} \operatorname{dist}\left(x, E_{n}\right)=0\right\}, \\
& \limsup _{n \rightarrow \infty} E_{n}:=\left\{x \in X: \liminf _{n \rightarrow \infty} \operatorname{dist}\left(x, E_{n}\right)=0\right\} .
\end{aligned}
$$

We shall use the following result due to Whyburn [28, (9.12)Theorem]:

Theorem 5.1. Assume $\left\{E_{n}\right\}$ is a sequence of connected sets satisfying that

(i) $\bigcup_{n \geq 1} E_{n}$ is precompact;

(ii) $\liminf _{n \rightarrow \infty} E_{n} \neq \emptyset$.

Then, $\limsup _{n \rightarrow \infty} E_{n}$ is nonempty, closed and connected.

As stated in Remark 3.1, we only have to prove Theorem 1.2(i) in the case $\int_{\Omega} a<0$.

5.1. Proof of assertion (i) in Theorems 1.1 and 1.2. When $\mathcal{B} u=\frac{\partial u}{\partial \mathbf{n}}$, we employ the following lemma, which concerns the direction of the bifurcation component $\mathcal{C}_{\varepsilon}$ at $(0,0)$, see [24, Theorem 5.1] for the proof.

Lemma 5.2. Let $\mathcal{B} u=\frac{\partial u}{\partial \mathbf{n}}$. Assume (1.9), (1.17) and $\int_{\Omega} a \neq 0$. Let $Z$ be any complement of $\langle 1\rangle$ in $W^{2, r}(\Omega)$. Then, for $\varepsilon>0$ small enough, the set $\{(\lambda, u)\}$ of nontrivial solutions of $\left(P_{\mathcal{B}, \varepsilon}\right)$ around $(0,0)$ is parametrized as

$$
(\lambda, u)=(\mu(s), s(1+z(s))),
$$


with $s \in\left(-s_{0}, s_{0}\right)$, for some $s_{0}>0$. Here $\mu:\left(-s_{0}, s_{0}\right) \rightarrow \mathbb{R}$ and $z:\left(-s_{0}, s_{0}\right) \rightarrow Z$ are continuous, and satisfy $\mu(0)=z(0)=0$. Therefore, $\mathcal{C}_{\varepsilon}$ is precisely described by $\left\{(\mu(s), s(1+z(s))): s \in\left[0, s_{0}\right)\right\}$ around $(0,0)$. Furthermore, the following holds:

$$
\lim _{s \rightarrow 0^{+}} \frac{\mu(s)}{s^{\sigma-1}}=-\varepsilon^{1-q} \frac{q g_{0} \int_{\Omega} b(x)}{f_{0} \int_{\Omega} a(x)} .
$$

In particular, under (1.16) and $\int_{\Omega} a<0$, the bifurcation of $\mathcal{C}_{\varepsilon}$ is subcritical at $(0,0)$.

Now, we consider the metric space $X:=\mathbb{R} \times V$ with the metric given by

$$
d((\lambda, u),(\mu, v)):=|\lambda-\mu|+\|u-v\|_{V} \quad \text { for }(\lambda, u),(\mu, v) \in \mathbb{R} \times V .
$$

From Corollary 4.6. if $\varepsilon \in(0,1]$, then the components $\mathcal{C}_{\varepsilon}^{ \pm}$of positive solutions of $\left(P_{\mathcal{B}, \varepsilon}\right)$, emanating from $\left(\lambda_{1, \varepsilon}^{ \pm}, 0\right)$, satisfy

$$
\mathcal{C}_{\varepsilon}^{ \pm} \subset\left\{(\lambda, u) \in \mathbb{R} \times V:|\lambda|+\|u\|_{V} \leq \bar{C}\right\}
$$

where $\bar{C}$ does not depend on $\varepsilon \in(0,1]$. This implies that $\mathcal{C}_{\varepsilon}^{ \pm}$are both bounded, and consequently, we deduce that $\mathcal{C}_{\varepsilon}^{-}=\mathcal{C}_{\varepsilon}^{+}$(cf. [1, Proposition 18.1]). Then, $\mathcal{C}_{\varepsilon}:=\mathcal{C}_{\varepsilon}^{ \pm}$ is nonempty and connected. In addition,

$$
(0,0) \in \liminf _{\varepsilon \rightarrow 0^{+}} \mathcal{C}_{\varepsilon},
$$

since $\lambda_{1, \varepsilon}^{ \pm} \rightarrow 0$ as $\varepsilon \rightarrow 0^{+}$. Moreover, by elliptic regularity, we obtain that

$$
\bigcup_{\varepsilon>0} \mathcal{C}_{\varepsilon} \text { is precompact. }
$$

Indeed, for any $\left\{\left(\lambda_{n}, u_{n}\right)\right\} \subset \bigcup_{\varepsilon>0} \mathcal{C}_{\varepsilon}$ we have that $\left(\lambda_{n}, u_{n}\right) \in \mathcal{C}_{\varepsilon_{n}}$ for some $\varepsilon_{n} \in$ $(0,1]$. From (5.2), we may assume that $\left\{\lambda_{n}\right\}$ is a convergent sequence. Using (5.2) again, we deduce that $u_{n} \in W^{2, r}(\Omega)$ are solutions of

$$
\begin{cases}-\Delta u_{n}=\lambda_{n} a(x)\left(u_{n}+\varepsilon_{n}\right)^{q-1} F\left(u_{n}\right)+b(x) g\left(u_{n}\right) & \text { in } \Omega, \\ u_{n}=0 & \text { on } \partial \Omega .\end{cases}
$$

In particular, using a bootstrap argument and the Sobolev embedding theorem, we deduce that $\left\|u_{n}\right\|_{C^{1+\theta}(\bar{\Omega})}$ is bounded, for some $\theta \in(0,1)$. The compact embedding $C^{1+\theta}(\bar{\Omega}) \subset C^{1}(\bar{\Omega})$ implies that $\left\{u_{n}\right\}$ has a convergent subsequence in $V$, as desired. Now, by (5.3) and (5.4), we may apply Theorem 5.1 to infer that $\mathcal{C}_{0}:=\limsup _{\varepsilon \rightarrow 0^{+}} \mathcal{C}_{\varepsilon}$ is non-empty, closed and connected in $\mathbb{R} \times V$. From (5.2), $\mathcal{C}_{0}$ is bounded in $\mathbb{R} \times V$. In addition, $\mathcal{C}_{0}$ is contained in the nonnegative solutions set of $\left(P_{\mathcal{B}}\right)$. Indeed, given $(\lambda, u) \in \mathcal{C}_{0}$, there exists $\left(\lambda_{n}, u_{n}\right) \in \mathcal{C}_{\varepsilon_{n}}$ such that $\varepsilon_{n} \rightarrow 0^{+}$ and $\left(\lambda_{n}, u_{n}\right) \rightarrow(\lambda, u)$ in $\mathbb{R} \times V$. Thus $u$ is a nonnegative weak solution of $\left(P_{\mathcal{B}}\right)$, and eventually, a nonnegative solution in $W^{2, r}(\Omega)$ by elliptic regularity.

Now, we show that $\mathcal{C}_{0}$ is nontrivial. By construction, we see that for $\varepsilon \rightarrow 0^{+}$, there exists a positive solution $u_{\varepsilon}$ of $\left(P_{\mathcal{B}, \varepsilon}\right)$ such that $\left(0, u_{\varepsilon}\right) \in \mathcal{C}_{\varepsilon}$. Indeed, we used Lemma 5.2 if $\mathcal{B} u=\frac{\partial u}{\partial \mathbf{n}}$. In this case, we observe from (5.1) that when (1.16) and $\int_{\Omega} a<0$ hold, the bounded component $\mathcal{C}_{\varepsilon}$ bifurcates subcritically at $(0,0)$, provided that $\varepsilon$ is small enough. This implies that $\mathcal{C}_{\varepsilon}$ cuts $\{(0, u): 0 \not \equiv u \geq 0\}$, 
and consequently, the desired assertion follows. Since $\left\|u_{\varepsilon}\right\|_{V} \leq \bar{C}$, it follows by combining elliptic regularity and standard compactness arguments as above that there exist $\varepsilon_{n} \rightarrow 0^{+}$and $u_{n}:=u_{\varepsilon_{n}}$ such that $u_{n}$ converges in $V$ to a nonnegative solution $u_{0}$ of $\left(P_{\mathcal{B}}\right)$ for $\lambda=0$. By definition, we have that $\left(0, u_{0}\right) \in \mathcal{C}_{0}$. From Proposition 4.8(ii), we infer that $u_{0}$ is nontrivial, and so, $u_{0} \gg 0$ by the strong maximum principle and Hopf's lemma. Assertion (i-1) has been now verified. We use Proposition 4.8(ii) again to deduce assertion (i-2).

Since $\mathcal{C}_{0}$ is nontrivial, we infer from Corollary 4.9 that $\mathcal{C}_{0}$ does not contain any $(\lambda, 0)$ with $\lambda \neq 0$. Assertion (1.6) has been verified.

Finally, we verify assertion (i-3). For $\rho>0$ and $\left(\lambda_{1}, u_{1}\right) \in \mathbb{R} \times V$, we set

$$
\begin{aligned}
& B_{\rho}\left(\left(\lambda_{1}, u_{1}\right)\right):=\left\{(\lambda, u) \in \mathbb{R} \times V:\left|\lambda-\lambda_{1}\right|+\left\|u-u_{1}\right\|_{V}<\rho\right\}, \\
& S_{\rho}\left(\left(\lambda_{1}, u_{1}\right)\right):=\left\{(\lambda, u) \in \mathbb{R} \times V:\left|\lambda-\lambda_{1}\right|+\left\|u-u_{1}\right\|_{V}=\rho\right\} .
\end{aligned}
$$

We note that $\overline{B_{\rho}\left(\left(\lambda_{1}, u_{1}\right)\right)}=B_{\rho}\left(\left(\lambda_{1}, u_{1}\right)\right) \cup S_{\rho}\left(\left(\lambda_{1}, u_{1}\right)\right)$.

Let $\Sigma_{\varepsilon}^{+}$and $\Sigma_{\varepsilon}^{-}$be closed connected subsets of $\left\{(\lambda, u) \in \mathcal{C}_{\varepsilon}: \lambda \geq 0\right\}$ and $\left\{(\lambda, u) \in \mathcal{C}_{\varepsilon}: \lambda \leq 0\right\}$, respectively, such that $\left(\lambda_{1, \varepsilon}^{ \pm}, 0\right),\left(0, u_{\varepsilon}^{ \pm}\right) \in \Sigma_{\varepsilon}^{ \pm}$for some positive solutions $u_{\varepsilon}^{ \pm}$of $\left(P_{\mathcal{B}}\right)$ for $\lambda=0$, see Figure 3. This is well defined thanks to Proposition 4.8 (ii). Since $\Sigma_{\varepsilon}^{ \pm} \subset \mathcal{C}_{\varepsilon}$, we observe that

$$
\Sigma_{0}^{ \pm}:=\limsup _{\varepsilon \rightarrow 0^{+}} \Sigma_{\varepsilon}^{ \pm} \subset \limsup _{\varepsilon \rightarrow 0^{+}} \mathcal{C}_{\varepsilon}=\mathcal{C}_{0}
$$

Repeating the argument above, Whyburn's topological approach yields that $\Sigma_{0}^{ \pm}$ are non-empty, closed and connected sets consisting of nonnegative solutions of $\left(P_{\mathcal{B}}\right)$ and such that $(0,0) \in \liminf _{\varepsilon \rightarrow 0^{+}} \Sigma_{\varepsilon}^{ \pm} \subset \Sigma_{0}^{ \pm}$. Proposition 4.8 (ii) tells us that $\left(0, u_{0}^{ \pm}\right) \in \Sigma_{0}^{ \pm}$, for some positive solutions $u_{0}^{ \pm}$of $\left(P_{\mathcal{B}}\right)$ with $\lambda=0$. It follows that $\Sigma_{0}^{ \pm} \neq\{(0,0)\}$, and by virtue of Corollary 4.9, that $\Sigma_{0}^{ \pm} \backslash\{(0,0)\}$ consists of nontrivial nonnegative solutions of $\left(P_{\mathcal{B}}\right)$.

By definition, $(\lambda, u) \in \Sigma_{0}^{+}$(respect. $\Sigma_{0}^{-}$) implies $\lambda \geq 0$ (respect. $\lambda \leq 0$ ). Lastly, by using Proposition 4.8(ii) again, there exists $\rho>0$ small such that $\Sigma_{0, \rho}^{ \pm}:=\Sigma_{0}^{ \pm} \cap \overline{B_{\rho}((0,0))}$ is closed and connected, and if $(\lambda, u) \in \Sigma_{0, \rho}^{ \pm} \backslash\{(0,0)\}$ then $\lambda \gtrless 0$. So, $\mathcal{C}_{0}^{ \pm}:=\Sigma_{0, \rho}^{ \pm}$have the desired properties.

5.2. Proof of assertion (ii) in Theorems 1.1 and 1.2. We consider the positivity of nontrivial nonnegative solutions of $\left(P_{\mathcal{B}}\right)$ with $f(s)=s^{q}, q \in(0,1)$. Let $\mathfrak{S}$ be the nontrivial nonnegative solutions set of $\left(P_{\mathcal{B}}\right)$, i.e.,

$$
\mathfrak{S}:=\left\{(\lambda, u) \in \mathbb{R} \times V: 0 \not \equiv u \geq 0 \text { solves }\left(P_{\mathcal{B}}\right)\right\} .
$$

Let $\mathfrak{C}$ be a nonempty connected subset of $\mathfrak{S}$, and let

$$
\mathfrak{C}^{\circ}:=\{(\lambda, u) \in \mathfrak{C}: u \gg 0\} .
$$

The following lemma ([15, Theorem 1.7]) provides us with a nonexistence result for nontrivial nonnegative solutions of (1.13), which plays an important role in our argument when $\mathcal{B} u=\frac{\partial u}{\partial \mathbf{n}}$ and $\int_{\Omega} a \geq 0$. 


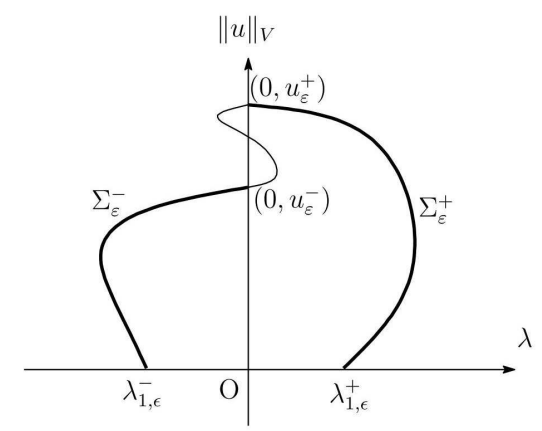

FIGURE 3 . The situations of $\Sigma_{\varepsilon}^{ \pm}$.

Lemma 5.3. Let $\mathcal{B} u=\frac{\partial u}{\partial \mathbf{n}}$. Assume $\left(H_{\psi}\right)$ with $\psi=a$. If $\int_{\Omega} a \geq 0$ then there exists $q_{a}^{*} \in(0,1)$ such that (1.13) has no nontrivial nonnegative solutions for any $q \in\left(q_{a}^{*}, 1\right)$.

We give now sufficient conditions for the positivity of the nontrivial nonnegative solutions on $\mathfrak{C}$ as follows. We recall that the sets $\mathcal{A}_{\mathcal{B}}^{ \pm a}$ are given by (1.14).

Proposition 5.4. Let $f(s)=s^{q}, q \in(0,1)$. Suppose (1.15), $\left(H_{\psi}\right)$ with $\psi= \pm a$, and the condition $b \geq 0$ in $\Omega$. Assume additionally $\int_{\Omega} a<0$ if $\mathcal{B} u=\frac{\partial u}{\partial \mathbf{n}}$. If

- $q \in \mathcal{A}_{\mathcal{B}}^{a} \cap \mathcal{A}_{\mathcal{B}}^{-a}$ (Dirichlet),

- $q \in \mathcal{A}_{\mathcal{B}}^{a} \cap\left(q_{-a}^{*}, 1\right)$ (Neumann),

where $q_{-a}^{*}$ is as in Lemma 5.3, then $\mathfrak{C}^{\circ}$ is open and closed in $\mathfrak{C}$. Consequently, $\mathfrak{C}^{\circ}=\mathfrak{C}$ if $\mathfrak{C}^{\circ} \neq \emptyset$.

Proof. It is straightforward that $\mathfrak{C}^{\circ}$ is open in $\mathfrak{C}$, since $u \gg 0$ for $(\lambda, u) \in \mathfrak{C}^{\circ}$. Next, we verify that $\mathfrak{C}^{\circ}$ is closed in $\mathfrak{C}$. Assume that $\left(\lambda_{n}, u_{n}\right) \in \mathfrak{C}^{\circ}$, and $\left(\lambda_{n}, u_{n}\right) \rightarrow$ $\left(\lambda_{0}, u_{0}\right) \in \mathfrak{C}$ in $\mathbb{R} \times V$. We shall show that $\left(\lambda_{0}, u_{0}\right) \in \mathfrak{C}^{\circ}$. We discuss the following three cases, in accordance with the sign of $\lambda_{0}$ :

(i) Case $\lambda_{0}>0$. We use the condition $q \in \mathcal{A}_{\mathcal{B}}^{a}$ to deduce the desired assertion. In this case, $\lambda_{n}>0$ for sufficiently large $n$. By the change of variables $v_{n}=\lambda_{n}^{-\frac{1}{1-q}} u_{n}$, we find that

$$
\begin{cases}-\Delta v_{n}=a(x) v_{n}^{q}+\lambda_{n}^{-\frac{1}{1-q}} b(x) g\left(\lambda_{n}^{\frac{1}{1-q}} v_{n}\right) & \text { in } \Omega, \\ \mathcal{B} v_{n}=0 & \text { on } \partial \Omega .\end{cases}
$$

Since $v_{n} \rightarrow v_{0}=\lambda_{0}^{-\frac{1}{1-q}} u_{0}$ in $V$, we find that $v_{0}$ is a nonnegative weak solution of the problem

$$
\begin{cases}-\Delta v=a(x) v^{q}+\lambda_{0}^{-\frac{1}{1-q}} b(x) g\left(\lambda_{0}^{\frac{1}{1-q}} v\right) & \text { in } \Omega, \\ \mathcal{B} v=0 & \text { on } \partial \Omega .\end{cases}
$$


In addition, $v_{0} \not \equiv 0$ in $\Omega_{+}^{a}$. Indeed, since (1.15) holds and $b \geq 0$, we see that $v_{n}$ is a supersolution of (1.13) which is positive in $\Omega$. So, condition $\left(H_{\psi}\right)$ with $\psi=a$ allows us to apply [15, Lemma 2.2], and deduce that there exist a ball $B \Subset \Omega_{+}^{a}$ and a continuous function $\psi$ on $\bar{B}$ such that $v_{n} \geq \psi>0$ in $B$. Passing to the limit, we have that $v_{0} \geq \psi$ in $B$, as desired.

By (1.15) and the condition $b \geq 0, v_{0}$ is a supersolution of (1.13), and $v_{0}>0$ in $B$. On the other hand, we can construct a nonnegative subsolution $\psi_{0}$ of (1.13) such that $\psi_{0} \not \equiv 0$ in $B, \psi_{0} \equiv 0$ in $\Omega \backslash B$, and $\psi_{0} \leq v_{0}$. The sub and supersolutions method provides us with a solution $v_{1}$ of (1.13) such that $\psi_{0} \leq v_{1} \leq v_{0}$, so that $v_{1} \gg 0$, since $q \in \mathcal{A}_{\mathcal{B}}^{a}$. Consequently, we conclude that $u_{0}=\lambda_{0}^{1 /(1-q)} v_{0} \gg 0$, as desired.

(ii) Case $\lambda_{0}<0$.

(ii-1) Case $\mathcal{B} u=u$ : We use the condition $q \in \mathcal{A}_{\mathcal{B}}^{-a}$ to deduce the desired assertion. In this case, $\lambda_{n}<0$ for sufficiently large $n$. Setting $\mu=-\lambda,\left(P_{\mathcal{B}}\right)$ turns into

$$
\begin{cases}-\Delta u=-\mu a(x) u^{q}+b(x) g(u) & \text { in } \Omega, \\ u=0 & \text { on } \partial \Omega .\end{cases}
$$

Set $\mu_{n}=-\lambda_{n}>0$, so that $v_{n}:=\mu_{n}^{-\frac{1}{1-q}} u_{n}$ satisfies

$$
\begin{cases}-\Delta v_{n}=-a(x) v_{n}^{q}+\mu_{n}^{-\frac{1}{1-q}} b(x) g\left(\mu_{n}^{\frac{1}{1-q}} v_{n}\right) & \text { in } \Omega, \\ v_{n}=0 & \text { on } \partial \Omega .\end{cases}
$$

Since $v_{n} \rightarrow v_{0}=\mu_{0}^{-\frac{1}{1-q}} u_{0}$ in $C_{0}^{1}(\bar{\Omega})$, we infer that $v_{0}$ is a nonnegative weak solution of the problem

$$
\begin{cases}-\Delta v=-a(x) v^{q}+\mu_{0}^{-\frac{1}{1-q}} b(x) g\left(\mu_{0}^{\frac{1}{1-q}} v\right) & \text { in } \Omega \\ v=0 & \text { on } \partial \Omega .\end{cases}
$$

By using the condition $q \in \mathcal{A}_{\mathcal{B}}^{-a}$, the rest of the argument is carried out similarly as in the previous case.

(ii-2) Case $\mathcal{B} u=\frac{\partial u}{\partial \mathbf{n}}$ : Under $q \in\left(q_{-a}^{*}, 1\right)$, we shall see that case $\lambda_{0}<0$ does not occur, using Lemma 5.3. We have $\lambda_{n}<0$ for $n$ sufficiently large. By setting $\mu=-\lambda,\left(P_{\mathcal{B}}\right)$ becomes

$$
\begin{cases}-\Delta u=-\mu a(x) u^{q}+b(x) g(u) & \text { in } \Omega, \\ \frac{\partial u}{\partial \mathbf{n}}=0 & \text { on } \partial \Omega .\end{cases}
$$

Setting $\mu_{n}=-\lambda_{n}>0$, and $v_{n}=\mu_{n}^{-\frac{1}{1-q}} u_{n}$, we find that

$$
\begin{cases}-\Delta v_{n}=-a(x) v_{n}^{q}+\mu_{n}^{-\frac{1}{1-q}} b(x) g\left(\mu_{n}^{\frac{1}{1-q}} v_{n}\right) & \text { in } \Omega, \\ \frac{\partial v_{n}}{\partial \mathbf{n}}=0 & \text { on } \partial \Omega .\end{cases}
$$


Since $v_{n} \rightarrow v_{0}=\mu_{0}^{-\frac{1}{1-q}} u_{0}$ in $C^{1}(\bar{\Omega})$, we infer that $v_{0}$ is a nonnegative weak solution of the problem

$$
\begin{cases}-\Delta v=-a(x) v^{q}+\mu_{0}^{-\frac{1}{1-q}} b(x) g\left(\mu_{0}^{\frac{1}{1-q}} v\right) & \text { in } \Omega \\ \frac{\partial v}{\partial \mathbf{n}}=0 & \text { on } \partial \Omega\end{cases}
$$

In addition, $v_{0} \not \equiv 0$ in $\Omega_{+}^{-a}$. Indeed, since (1.15) holds, and $b \geq 0$, we see that $v_{n}$ is a positive supersolution of

$$
\begin{cases}-\Delta v=-a(x) v^{q} & \text { in } \Omega, \\ \frac{\partial v}{\partial \mathbf{n}}=0 & \text { on } \partial \Omega,\end{cases}
$$

so that, by [15, Lemma 2.2], there exists a ball $B \Subset \Omega_{+}^{-a}$ and a continuous function $\psi$ on $\bar{B}$ such that $v_{n} \geq \psi>0$ in $B$. Passing to the limit, we obtain $v_{0} \geq \psi$ in $B$, as desired.

Now, we see that $v_{0}$ is also a nonnegative supersolution of (5.6) such that $v_{0}>0$ in $B$. Since we can construct a nonnegative subsolution $\psi_{0}$ of $\left(\underline{5.6}\right.$ ) such that $\psi_{0} \not \equiv 0$ in $B, \psi_{0} \equiv 0$ in $\Omega \backslash B$, and $\psi_{0} \leq v_{0}$, the sub and supersolutions method provides us with a solution $v_{1}$ of (5.6) such that $\psi_{0} \leq v_{1} \leq v_{0}$ on $\bar{\Omega}$. So, $v_{1}$ is nontrivial and nonnegative. However, this contradicts Lemma 5.3. since $\int_{\Omega}(-a)>0$ and $q_{-a}^{*}<q<1$.

(iii) Case $\lambda_{0}=0$. In this case, $u_{0}$ solves the problem

$$
\begin{cases}-\Delta u_{0}=b(x) g\left(u_{0}\right) & \text { in } \Omega \\ \mathcal{B} u_{0}=0 & \text { on } \partial \Omega\end{cases}
$$

Since $u_{0}$ is nontrivial and nonnegative, the strong maximum principle and Hopf's lemma yield that $u_{0} \gg 0$, as desired.

Lastly, since $\mathfrak{C}$ is connected, we conclude that $\mathfrak{C}^{\circ}=\mathfrak{C}$ if $\mathfrak{C}^{\circ} \neq \emptyset$.

Introducing the following growth condition on $g$ :

$$
0<\lim _{s \rightarrow \infty} \frac{g(s)}{s^{p}}<\infty \text { for some } p>1 \text {, where } p<\frac{N+1}{N-1} \text { if } N>2,
$$

we can deduce that $\mathfrak{C}^{\circ} \neq \emptyset$, as shown (i) and (ii) below.

\section{Remark 5.5.}

(i) If $\left(0, u_{0}\right) \in \mathfrak{C}$ with $u_{0} \not \equiv 0$, then $u_{0} \gg 0$, i.e., $\left(0, u_{0}\right) \in \mathfrak{C}^{\circ}$. Indeed, this is a direct application of the strong maximum principle and Hopf's lemma.

(ii) Assume (1.3) and $\left(H_{\psi}\right)$ with $\psi=a$. Assume also (1.12) if $\mathcal{B} u=u$; and (5.7) and $\int_{\Omega} a<0$ if $\mathcal{B} u=\frac{\partial u}{\partial \mathbf{n}}$. Let $b \equiv 1$ and $q \in \mathcal{A}_{\mathcal{B}}^{a}$. If there exist $\left(\lambda_{n}, u_{n}\right) \in \mathfrak{C}$ with $\lambda_{n} \rightarrow 0^{+}$, then $u_{n} \gg 0$ for sufficiently large $n$, i.e., $\left(\lambda_{n}, u_{n}\right) \in \mathfrak{C}^{\circ}$ for such $n$. This is an immediate consequence of [15, Theorem 4.1, Theorem 4.5].

(iii) When $\mathcal{B} u=\frac{\partial u}{\partial \mathbf{n}}$, Proposition 5.4 is valid for $\int_{\Omega} a>0$, where we now assume $q \in \mathcal{A}_{\mathcal{B}}^{-a} \cap\left(q_{a}^{*}, 1\right)$ instead of $q \in \mathcal{A}_{\mathcal{B}}^{a} \cap\left(q_{-a}^{*}, 1\right)$. Indeed, when $\int_{\Omega} a>0$, the case $\lambda_{0}>0$ does not occur, based on Lemma 5.3. whereas the case $\lambda_{0}<0$ 
is verified as in the proof of Proposition [5.4, using $\lambda a(x)=(-\lambda)(-a(x))$ and relying on [15, Lemma 2.2].

(iv) Proposition 5.4 and item (ii) hold more generally in the framework $\mathbb{R} \times$ $C(\bar{\Omega})$, which can been seen by using elliptic regularity.

Proof of assertion (ii) in Theorem 1.1. We note from Remark 2.2(iii) that (1.15) holds in case (b) of Theorem 1.1. Based on the result stated in Remark 5.5(i), this assertion is verified by a direct application of Proposition 5.4

Proof of assertion (ii) in Theorem 1.2, Based on the result stated in Remark 5.5(ii), this assertion is straightforward from Proposition 5.4 and Remark 5.5(iv). Indeed, we don't need to assume $\left(H_{\psi}\right)$ with $\psi=-a$ for applying Proposition 5.4 to the loop $\mathcal{C}_{*}$ given in Theorem 1.2(ii), since it lies in $\lambda \geq 0$ (see Figure 1(ii)). Note that the condition $\left(H_{\psi}\right)$ with $\psi=-a$ is used only for case (ii) in the proof of Proposition 5.4

\section{REFERENCES}

[1] H. Amann, Fixed point equations and nonlinear eigenvalue problems in ordered Banach spaces, SIAM Rev. 18, (1976), 620-709.

[2] H. Amann and J. López-Gómez, A priori bounds and multiple solutions for superlinear indefinite elliptic problems, J. Differential Equations 146, (1998), 336-374.

[3] A. Ambrosetti, H. Brezis and G. Cerami, Combined effects of concave and convex nonlinearities in some elliptic problems, J. Funct. Anal. 122, (1994), 519-543.

[4] K. J. Brown, Local and global bifurcation results for a semilinear boundary value problem, J. Differential Equations 239, (2007), 296-310.

[5] S. Cano-Casanova, Compact components of positive solutions for superlinear indefinite elliptic problems of mixed type, Topol. Methods Nonlinear Anal. 23, (2004), 45-72.

[6] S. Cano-Casanova, J. López-Gómez and M. Molina-Meyer, Isolas: compact solution components separated away from a given equilibrium curve, Hiroshima Math. J. 34 (2004), 177-199.

[7] S. Cano-Casanova, J. López-Gómez and M. Molina-Meyer, Bounded components of positive solutions of nonlinear abstract equations, translated from Ukr. Mat. Visn. 2 (2005), 38-51, Ukr. Math. Bull. 2 (2005), 39-52.

[8] S. Cingolani and J. L. Gámez, Positive solutions of a semilinear elliptic equation on $\mathbf{R}^{N}$ with indefinite nonlinearity, Adv. Differential Equations 1, (1996), 773-791.

[9] M. G. Crandall and P. H. Rabinowitz, Bifurcation from simple eigenvalues, J. Funct. Anal. 8, (1971), 321-340.

[10] D. G. de Figueiredo, J-P. Gossez and P. Ubilla, Local superlinearity and sublinearity for indefinite semilinear elliptic problems, J. Funct. Anal. 199, (2003), 452-467.

[11] D. G. de Figueiredo, J-P. Gossez and P. Ubilla, Multiplicity results for a family of semilinear elliptic problems under local superlinearity and sublinearity, J. Eur. Math. Soc. 8, (2006), 269-286.

[12] J. Fleckinger, J. Hernández and F. de Thélin, Existence of multiple principal eigenvalues for some indefinite linear eigenvalue problems, Boll. Unione Mat. Ital. Sez. B Artic. Ric. Mat. (8) $\mathbf{7},(2004), 159-188$.

[13] B. Gidas and J. Spruck, A priori bounds for positive solutions of nonlinear elliptic equations, Comm. Partial Differential Equations 6, (1981), 883-901. 
[14] D. Gilbarg and N. S. Trudinger, Elliptic partial differential equations of second order, Reprint of the 1998 edition, Classics in Mathematics. Springer-Verlag, Berlin, 2001.

[15] U. Kaufmann, H. Ramos Quoirin and K. Umezu, Positivity results for indefinite sublinear elliptic problems via a continuity argument, J. Differential Equations 263, (2017), 4481-4502.

[16] U. Kaufmann, H. Ramos Quoirin and K. Umezu, Positive solutions of an elliptic Neumann problem with a sublinear indefinite nonlinearity, NoDEA Nonlinear Differ. Equ. Appl. (2018) 25:12.

[17] U. Kaufmann, H. Ramos Quoirin and K. Umezu, A curve of positive solutions for an indefinite sublinear Dirichlet problem, preprint. arXiv:1709.04822

[18] J. López-Gómez, Spectral theory and nonlinear functional analysis. Research Notes in Mathematics 426, Chapman \& Hall/CRC, Boca Raton, FL, 2001.

[19] J. López-Gómez, Linear second order elliptic operators, World Scientific Publishing Co. Pte. Ltd., Hackensack, NJ, 2013.

[20] J. López-Gómez and M. Molina-Meyer, Bounded components of positive solutions of abstract fixed point equations: mushrooms, loops and isolas, J. Differential Equations 209, (2005), 416-441.

[21] J. López-Gómez, M. Molina-Meyer and A. Tellini, The uniqueness of the linearly stable positive solution for a class of superlinear indefinite problems with nonhomogeneous boundary conditions, J. Differential Equations 255, (2013), 503-523.

[22] J. López-Gómez and A. Suárez, Combining fast, linear and slow diffusion, Topol. Methods Nonlinear Anal. 23 (2004), 275-300.

[23] P. H. Rabinowitz, Some global results for nonlinear eigenvalue problems, J. Functional Analysis 7, (1971), 487-513.

[24] H. Ramos Quoirin and K. Umezu, An indefinite concave-convex equation under a Neumann boundary condition I, Israel J. Math. 220, (2017), 103-160.

[25] H. Ramos Quoirin and K. Umezu, An indefinite concave-convex equation under a Neumann boundary condition II, Topol. Methods Nonlinear Anal. 49, (2017), 739-756.

[26] H. Ramos Quoirin and K. Umezu, A loop type component in the non-negative solutions set of an indefinite elliptic problem, Comm. Pure Appl. Anal. 17, (2018), 1255-1269.

[27] K. Umezu, Bifurcation approach to a logistic elliptic equation with a homogeneous incoming flux boundary condition, J. Differential Equations 252, (2012), 1146-1168.

[28] G. T. Whyburn, Topological analysis. Second edition, Princeton Mathematical Series, No. 23, Princeton University Press, Princeton, N.J., 1964.

U. KaUfmann

Famaf, Universidad Nacional de Córdoba, (5000) Córdoba, Argentina

E-mail address: kaufmann@mate.uncor.edu

H. RAMOS QUOIRIN

Universidad de Santiago de Chile, Casilla 307, Correo 2, Santiago, Chile

E-mail address: humberto.ramos@usach.cl

K. UMEZU

Department of Mathematics, Faculty of Education, Ibaraki University, Mito 310-8512, JAPAN

E-mail address: kenichiro.umezu.math@vc.ibaraki.ac.jp 